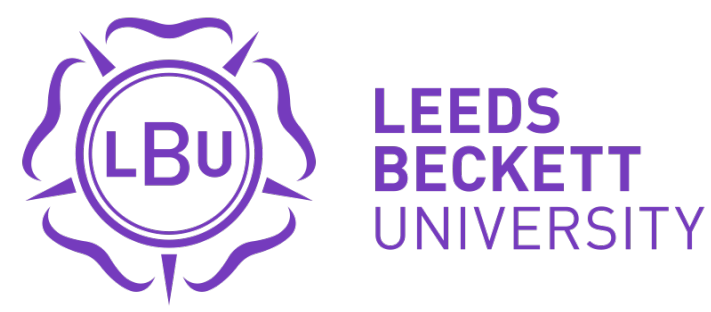

Citation:

Cassano, A and Marchese Robinson, RL and Palczewska, A and Puzyn, T and Gajewicz, A and Tran, L and Manganelli, S and Cronin, MTD (2016) Comparing the CORAL and Random Forest approaches for modelling the in vitro cytotoxicity of silica nanomaterials. Alternatives to Laboratory Animals, 44 (6). pp. 533-556. ISSN 0261-1929 DOI: https://doi.org/10.1177/026119291604400603

Link to Leeds Beckett Repository record:

https://eprints.leedsbeckett.ac.uk/id/eprint/5729/

Document Version:

Article (Accepted Version)

Creative Commons: Attribution-Noncommercial-No Derivative Works 4.0

The aim of the Leeds Beckett Repository is to provide open access to our research, as required by funder policies and permitted by publishers and copyright law.

The Leeds Beckett repository holds a wide range of publications, each of which has been checked for copyright and the relevant embargo period has been applied by the Research Services team.

We operate on a standard take-down policy. If you are the author or publisher of an output and you would like it removed from the repository, please contact us and we will investigate on a case-by-case basis.

Each thesis in the repository has been cleared where necessary by the author for third party copyright. If you would like a thesis to be removed from the repository or believe there is an issue with copyright, please contact us on openaccess@leedsbeckett.ac.uk and we will investigate on a case-by-case basis. 


\section{Comparing the CORAL and Random Forest approaches for modelling the in vitro 2 cytotoxicity of silica nanomaterials}

3 Antonio Cassano ${ }^{\mathrm{a}}$, Richard L. Marchese Robinson ${ }^{\mathrm{a}}$, Anna Palczewska ${ }^{\mathrm{b}}$, Tomasz Puzyn ${ }^{\mathrm{c}}$, Agnieszka Gajewicz ${ }^{\mathrm{c}}$, $4 \quad$ Lang Tran ${ }^{\mathrm{d}}$, Serena Manganellie, Mark T. D. Cronin ${ }^{* a}$

$5 \quad{ }^{a}$ School of Pharmacy and Biomolecular Sciences, Liverpool John Moores University, Byrom Street, Liverpool, L3 $63 A F$, England.

$7 \quad{ }^{b}$ University of Leeds, School of Geography, Leeds, England.

8 'Laboratory of Environmental Chemistry, University of Gdansk, Wita Stwosza 63, 80-308 Gdansk, Poland.

$9 \quad{ }^{d}$ Institute of Occupational Medicine, Edinburgh, Midlothian, Scotland. ${ }^{e}$ IRCSS-Istituto di Ricerche Farmacologiche Mario Negri, Via Giuseppe La Masa, 19, 20156, Milan, Italy.

\section{Summary}

Nanotechnology is one of the most important technological developments of the twenty-first century. In silico methods such as quantitative structure-activity relationships (QSARs) to predict toxicity promote the safe-bydesign approach for the development of new materials, including nanomaterials. In this study, a set of cytotoxicity experimental data corresponding to 19 data points for silica nanomaterials was investigated to compare the widely employed CORAL and Random Forest approaches in terms of their usefulness for developing so-called "nanoQSAR" models. "External" leave-one-out cross-validation (LOO) analysis was performed to validate the two different approaches. An analysis of variable importance measures and signed feature contributions for both algorithms was undertaken in order to interpret the models developed. CORAL showed a more pronounced difference between the average coefficient of determination $\left(\mathrm{R}^{2}\right)$ between training and LOO $(0.83$ and 0.65 for training and LOO respectively) compared to Random Forest ( 0.87 and 0.78 without bootstrap sampling, 0.90 and 0.78 with bootstrap sampling), which may be due to overfitting. The aspect ratio and zeta potential from amongst the nanomaterials' physico-chemical properties were found to be the two most important variables for the Random Forest and the average feature contributions calculated for the corresponding descriptors were consistent with the clear trends observed in the dataset: less negative zeta potential values and lower aspect ratio values were associated with higher cytotoxicity. In contrast, CORAL failed to capture these trends.

Keywords: pseudo-SMILES; nano-QSAR; CORAL software; Random Forest; silica nanoparticle; variable importance; feature contribution Byrom Street, Liverpool, L3 3AF, England. Email address: M.T.Cronin@ljmu.ac.uk 


\section{Introduction}

Nanotechnology, which may be defined as the technological application of engineered nanomaterials [1], is considered to be one of the most important technological developments of the $21^{\text {st }}$ century [2, 3] so much so that the term "nano-revolution" has been used to describe the growth of this industry [4]. Nanotechnology is able to produce engineered nanomaterials having new or enhanced physico-chemical properties compared to the bulk material. However, some of these properties, e.g. high surface area to volume ratio, are potentially dangerous to humans [5-9]. In silico methods (e.g. (Q)SAR, grouping and read-across) promote the safe-by-design approach for the development of new nanomaterials by studying the relationship between the nanomaterials' "structures" and their biological effects [10, 11]. Since nanomaterials are complex [12], typically polydisperse, particulate materials, the concept of a "structure" in this context should not be confused with a single molecular structure but rather a description of the nanomaterial in terms of its measurable physico-chemical characteristics $[9,13]$ such as the composition of different components, aspect ratio etc. In this regard, the development of nanomaterial quantitative structure-activity relationships ("nano-QSAR") may offer an effective alternative to experimental testing, since they may enable the prediction of (eco)toxicological effects of nanomaterials based on a knowledge of their chemical composition and, where necessary, other physico-chemical properties [14-16]. QSAR models can be classified as linear or non-linear depending on whether they were developed using a linear method, such as a multiple linear regression [17, 18], or a non-linear methods, such as support vector machines in combination with a non-linear kernel function [19, 23] or Random Forest [24, 25]. The aim of this study was to evaluate different approaches to build nano-QSAR models for a dataset comprising 19 cytotoxicity experimental data points for silica nanomaterials. We focused on silica nanomaterials mainly because of the availability of a novel experimental dataset for nanomaterials with a silica core and due to the widespread use of silica based nanomaterials in consumer products (http://www.nanotechproject.org/cpi/browse/nanomaterials/silicon-dioxide/). In this work, a comparison was made between two commonly used approaches to develop QSAR and nano-QSAR models: the linear approach implemented in the CORelation And Logic (CORAL) program, which optimises a (linear) regression model using a Monte Carlo search procedure [26], and Breiman's non-linear Random Forest algorithm [24, 25], implemented in the $\mathrm{R}$ randomForest package [27]. Our motivation to focus on these two modelling approaches reflects the fact that these have been used to build QSAR/QSPRs (and nanoQSAR/QSPRs) for a variety of different datasets, as illustrated in the number of publications summarised in the Supplementary Information (SI); for instance, 28 and 21 articles describing QSAR/QSPRs and 
nano-QSAR/QSPRs studies using the COARL and Random Forest approaches respectively, were published in 2015. (Quantitative structure-property relationships, or QSPRs, are analogous to QSARs, but aim to predict non-biological properties.) However, to the best of our knowledge, these algorithms have never previously been compared. Indeed, Random Forest has only twice before been used to model nanomaterial effects $[28,29]$. Hence, this investigation serves as a timely comparison of two widely employed QSAR modelling approaches on a suitable dataset. In addition to comparing their predictive performance, we performed a comparison in terms of model interpretability between a linear (CORAL) and a non-linear (Random Forest) approach. In other words, the ability of the two selected approaches to describe the toxicological trends of this dataset was evaluated.

\section{Materials and Methods}

\subsection{Experimental data}

The experimental data used to develop the models correspond to a subset extracted from the dataset generated during the MODENA COST Initiative (MODENA TD1204 COST ACTION dataset, http://www.modena-cost.eu/Home.aspx). This dataset is provided in Table 1 and it is available electronically in the SI. The dataset consists of 19 in vitro WST-1 cytotoxicity experimental data points for uncoated silica nanomaterials. Briefly, WST-1 is a colorimetric assay for assessing cell metabolic activity which is similar to the MTT assay, but which offers certain experimental advantages [30, 31]. The changes in metabolic activity measured using the WST-1 assay are considered a proxy for changes in cell viability [32]. The data used in this work consist of 19 values for the negative logarithm of the $\mathrm{EC}_{25}$ i.e. the concentration level which induces $25 \%$ of maximum response above the baseline after a given treatment time. For modelling, nanomaterial concentrations, hence the corresponding $\mathrm{EC}_{25}$ values, were expressed as surface area of nanomaterial per millilitre (i.e. $\mathrm{mm}^{2} / \mathrm{ml}$ ), in keeping with guidance from the Organisation for Economic Co-operation and Development [33]. Cytotoxicity data range from -1.299 to 0.483 , with no values between -0.822 and -0.394 i.e. the data cluster at low and high activities as shown in Figure 1. Furthermore, from the original dataset we selected five variables based on our expert judgement expected to explain variability in these activities: treatment time and cell type are related to the experimental conditions adopted in the assay protocol, whereas average size, aspect ratio and zeta potential are measured physico-chemical properties of silica nanomaterials. Specifically, since CORAL is only able to handle a maximum of five variables, less significant descriptors were discarded. The full list of descriptors can be found in the SI. 


\subsection{Evaluation approach}

We adopted an "external" leave-one-out (LOO) cross-validation technique as a method to validate the considered modelling approaches. In brief, LOO is a special case of cross-validation [34-36], where the number of folds equals the number of instances in the data set. In other words, the learning algorithm is applied once for each instance, using all other instances as a training set and using the selected instance as a single-item test set. To this respect, for the dataset used in this work which comprises 19 instances, both CORAL and Random Forest algorithms were applied 19 times over all the instances in the dataset, each time considering 18 instances as training set and the remaining one as a test set in order to generate a given set of LOO results. (For both methods, five sets of LOO results were obtained as explained below.) By "external" LOO, we mean that all model development - including selection of descriptors and algorithm parameters or "hyperparameters" - was carried out exclusively using each LOO training set in turn i.e. the biological activity of the correspond test instance was not considered, to remove this potential source of optimistic bias from the results [37-39]. The coefficient of determination $\left(\mathrm{R}^{2}\right)$ and the root mean square error (RMSE) were here used as statistics for comparing the two approaches, according to the equations (1) and (2) [36], based on two $n$ value vectors $y_{1} \ldots y_{n}$ and $f_{1} \ldots f_{n}$ which are associated with the experimental and predicted values respectively. N.B. (a) As the dataset comprised 19 instances, $\mathrm{n}=18$ for training sets whereas $n=19$ for LOO. (b) In equations (1) and (2), and all subsequent equations in this manuscript, the "-" character indicates the arithmetic mean (or "average") value over all the elements of a vector.

Coefficient of determination $=\mathbf{R}^{2}=1-\frac{\sum_{i=1}^{n}\left(y_{i}-f_{i}\right)^{2}}{\sum_{i=1}^{n}\left(y_{i}-\bar{y}\right)^{2}}$

(1)

Root mean square error $=\mathbf{R M S E}=\sqrt{\frac{\sum_{i=1}^{n}\left(y_{i}-f_{i}\right)^{2}}{n}}$

We performed the LOO validation technique five times, since the CORAL and Random Forest algorithm s employ random selections during the model building phase, in order to obtain a more robust estimate of the performance of these methods. We selected five different seeds for each repetition with the Random Forest algorithm. We further selected five different dataset partitions for each repetition with CORAL. (Each time the CORAL software is run, it automatically generates a random seed that cannot be set by 
the end user.) Each dataset partition corresponds to partitioning of a given LOO training set, following removal of a single test set instance for "external" LOO validation, to yield an internal "test set" for hyperparameter selection. Repeating modelling with CORAL five times in this fashion is broadly in keeping with the recommended procedure for CORAL model optimisation and robustness evaluation [4042]. Further discussion of the CORAL hyperparameters which were optimised is presented under "CORAL modelling". The average $(\overline{\mathbf{a}})$, standard deviation (s) and standard error of the mean (SE) for the LOO $\mathrm{R}^{2}$ and RMSE statistics across the five different repetitions were calculated as shown in equations (3), (4) and (5), considering the three general formulas based on a vector of $m$ values i.e. $a_{1}, a_{2}, \ldots, a_{m}$. N.B. Here, $m=19$ for training set results averaged for a single seed (or CORAL training set split), or 95 $(19 \times 5)$ for "global" training set results, whereas $m=5$ for LOO results.

Average $=\overline{\mathbf{a}}=\frac{1}{\mathrm{~m}} \sum_{\mathrm{i}=1}^{\mathrm{m}} \mathbf{a}_{\mathbf{i}}$

Standard deviation $=\mathbf{s}=\sqrt{\frac{\sum(\mathbf{a}-\bar{a})^{2}}{(\mathbf{m}-\mathbf{1})}}$

Standard error of the mean $=\mathrm{SE}=\frac{s}{\sqrt{m}}$

\subsection{Descriptor calculations}

As is further explained under "CORAL modelling", continuous numeric properties, such as zeta potential, were converted into binary descriptors corresponding to labels applied to specific ranges: each descriptor took a value of 1 (or 0 ) if the corresponding property value for a given instance was inside (or outside) of this range. In the case of the discrete qualitative variable "Cell Type", each value was converted into a binary descriptor: the descriptor took the value 1 (or 0) if the "Cell Type" for a given instance matched (or did not match) the value associated with that descriptor. This was necessary since, as is explained under "CORAL modelling", the CORAL algorithm can only work with binary descriptors. These binary descriptors were used for both CORAL and Random Forest modelling. For the CORAL software, these descriptors were represented implicitly i.e. the presence of a corresponding label in a pseudo-SMILES (see "CORAL modelling") denotes a descriptor value of 1. For the implementation of Random Forest 
used in the current work (see "Random Forest modelling"), these binary descriptors were represented as an explicit bit-string.

\subsection{Correlated descriptors}

We tested the influence of correlated descriptors on model results for both CORAL and Random Forest approaches by generating two versions of the original dataset, as shown in Table 2 . In one case, after the binary splitting was applied to each continuous numeric variable, a label was assigned to each of the generated value ranges, which translates into two perfectly correlated descriptors for a given continuous numeric variable. For instance, by splitting the "Treatment Time" variable into 24 and 48 hours, we generated two labels, namely "A" and "B", which refer to the 24 and 48 hours' exposure respectively, in the in vitro model. This results in the generation of two perfectly correlated descriptors, since they are mutually exclusive. Specifically, when the " $A$ " label is applicable (i.e. the " $A$ " descriptor value is 1), the " $\mathrm{B}$ " label must not be applicable (i.e. the " $\mathrm{B}$ " descriptor value is 0 ), and vice-versa, according to the fact that, a single experimental result can only be associated with a single "Treatment Time" value. In the second case, after the splitting of the continuous numeric variables, only one of the ranges was assigned a label and, hence, a corresponding binary descriptor. As a result, perfectly correlated descriptors were removed. For the sake of brevity, even though the second approach does use correlated descriptors for the cell line variable, throughout this paper results obtained "with correlated descriptors" refer to the first approach i.e. two labels for each continuous numeric variable, whereas results obtained "without correlated descriptors" refer to the second approach. In the main text of this paper, only results obtained without correlated descriptors were presented, with results obtained with correlated descriptors presented in the SI for comparison.

\subsection{CORAL modelling}

In this work, the Monte Carlo algorithm implemented in the CORAL software (version: December 17, 2014 for Microsoft Windows, available at http://www.insilico.eu/coral/) was used as a tool for developing linear nano-QSAR models, taking into account both the information derived from the nanomaterials' physico-chemical properties (e.g. zeta potential) and the experimental conditions (e.g. cell type). Specifically, after we downloaded the zipped file from the aforementioned website con taining the binary executable files, we executed the CORAL.exe binary file included in the folder “CORALSEAIMyCORALSEAIREGRESSION” to perform the modelling. In keeping with earlier work, we generated a "pseudo-SMILES" string for each instance which represented both information related to 
particular experimental conditions and nanomaterial properties [40]. In more detail, with this particular approach, all the eclectic information is used for modelling, with the endpoint of interest being a function of both the nanomaterials' physico-chemical properties and experimental conditions. Pseudo-SMILES character strings were derived as shown in Table 2 . When used to build linear models, as in the current work, the CORAL algorithm effectively treats each character (or label) in the pseudo-SMILES strings as a binary descriptor which takes a value of 1 (or 0 ) if the character is present (or absent) for a given instance $[40,43]$. The manner in which predictions are obtained, based on the values of these descriptors for a given instance, is further explained when discussing "Variable importance" below (see equations 6 and 7). Table 2 shows the selected labelling approaches with and without correlated descriptors, but we reported in the main text only results obtained without correlated descriptors (leave-one-out results obtained with correlated descriptors can be found in the SI). For the current dataset, after removing correlated descriptors, pseudo-SMILES labels were generated as follows. The information on the cell type was coded with the 'C', 'D', 'E', 'F' and 'G' characters for the 16HBE, A549, HaCaT, NRK-52E and THP-1 cell types, respectively. For all numeric descriptors in the dataset, a "binary split" was performed i.e. numeric values beyond some threshold were assigned a label and values before that threshold were not, thus avoiding incorporating perfectly correlated descriptors. Specifically, for the treatment time descriptor, a label 'A' was assigned if the exposure time was 24 hours, whereas no label was assigned if the exposure time was 48 hours. For each of the three properties related to the nanomaterial physico-chemical properties, namely average size, aspect ratio and zeta potential, a binary split of the values was applied based on the median value for the dataset, with the rationale of having a similar number of instances in a given range for each property. (N.B. The odd number of instances - i.e. 19 - in the dataset meant that the number of instances in each range, for each binary split, could not be perfectly equal and the ranges are expressed in terms of the values just beyond the median for aspect ratio and zeta potential.) The thresholds used for the splits were 27.5 (no label for values below or equal to the threshold, 'I' for values above it), 1.0 (no label for values equal to the threshold, 'K' for values above it) and -32.0 (no label for values below the threshold, ' $\mathrm{L}$ ' for values equal to or greater than it) for the average size, aspect ratio and zeta potential, respectively. In earlier work with CORAL [40-42], the authors developed five different splits of the same dataset in order to check whether the developed models were obtained by chance. According to the recommended CORAL optimisation strategy, we selected the best hyperparameter values (i.e. $\mathrm{N}=$ number of epochs, $\mathrm{T}=$ threshold) using the model performance on a 
212 subset of the dataset, which is called a "test set" in the CORAL software documentation, and then we

213 predicted the single item "external" test set in a separate step after the model was built. For each LOO

214 training set, modelling was repeated five times, via splitting the training set to yield an internal "test set"

215 for hyperparameter selection, five times. More details on the application of the CORAL software to this

216 dataset are reported in the SI, including full details of the five different LOO training set partitions used

217 for hyperparameter selections. (See "Details on the CORAL software settings and optimisation" in the

218 SI.)

219

220

\subsection{Random Forest modelling}

221

Random Forest is an ensemble learning method for both classification and regression which operates by building a multitude of decision trees, providing as output the class which represents the majority prediction, for classification problems, or the average prediction, for regression problems, of the individual trees $[24,25]$. Each decision tree is grown using an independent random sample of the instances in the training set, with the descriptors considered for splitting each node being independently sampled from the total. In the current work, both bootstrap sampling of the training set, i.e. sampling of $\mathrm{N}$ from $\mathrm{N}$ with replacement, and sampling without replacement were considered. The results presented in the main text were obtained without bootstrap sampling, with results obtained with bootstrap sampling being reported in SI. Whilst bootstrap sampling is typically used [25, 27] it is not currently possible to calculate feature contributions (see the "Feature contribution analysis" section) with the available software [44] if bootstrap sampling is used. The results in the SI show that, for this dataset, the model performance and standard variable importance measures (see the "Variable importance" section) are very similar with both types of sampling. In this work, we used the Random Forest algorithm implemented in the randomForest R package (version 4.6-12) [27], with the default values for the algorithm "hyperparameters" i.e. number of trees to grow (ntree) equal to 500 and the number of descriptors randomly sampled at each split (mtry) equal to the total number of descriptors in the dataset divided by three (for regression problems) as explained in the randomForest package documentation. The experimental data used for Random Forest modelling were the same as for the CORAL software. The binary descriptors implicitly encoded in the pseudo-SMILES strings created for the CORAL software were explicitly represented for modelling using the randomForest package i.e. a "1" value was assigned each time a specific label was present whereas a "0" value was assigned each time the label was absent in the considered pseudo-SMILES. Using this procedure, an explicit bit string was built for each pseudo-SMILES, as shown in Table 3. As per modelling 
with CORAL, with the Random Forest algorithm 95 models were developed with a given sampling protocol i.e. 19 models for each LOO training set and all modelling on a given training set was repeated five times to take account of the random sampling inherent to building models with Random Forest or CORAL. This process was repeated twice with two different sampling protocols: with simple sampling, without replacement, or bootstrap sampling i.e. the replace argument of the randomForest() function was set to FALSE and TRUE respectively. Hence, 190 Random Forest models were built in total with or without correlated descriptors. (It should be reiterated that only results "without correlated descriptors", meaning without perfectly correlated descriptors, without bootstrap sampling are presented in the main text.)

\subsection{Variable importance}

\subsubsection{CORAL}

In the current work, we selected the additive scheme of the CORAL software which computes a so-called "optimal descriptor" (DCW) as the sum of correlation weights associated with the labels present in the pseudo-SMILES strings [40, 43], according to equation (6).

$$
\operatorname{DCW}\left(\text { Threshold, } \mathrm{N}_{\mathrm{epoch}}\right)_{i}=\sum_{k=1}^{5} \mathrm{Cw}_{k} \times \mathrm{SA}_{\mathrm{k}, \mathrm{i}}
$$

N.B. In equation (6), $\mathrm{SA}_{\mathrm{k}, \mathrm{i}}$ takes the value 1 (or 0 ) if the corresponding pseudo-SMILES label is present (or absent) in an instance (i) i.e. the correlation weights $\left(\mathrm{Cw}_{\mathrm{k}}\right)$ are summed over all labels present in a given instance. In order to understand the relationship between the correlation weights and the final predicted value, it is important to note that the so-called "optimal descriptor" is used to calculate the predicted value for the endpoint using a one variable linear equation, as shown in equation (7).

$$
\text { Prediction }_{i}=\mathrm{C}_{0}+\mathrm{C}_{1} * \operatorname{DCW}\left(\text { Threshold, } \mathrm{N}_{\mathrm{epoch}}\right)_{i}
$$

Hence, it can be seen that the correlation weights are essentially scaled values of (i.e. are directly proportional to) the coefficients of the binary descriptors in the final linear model developed using CORAL. In order to make a comparison between CORAL and the standard Random Forest methods for variable importance, we calculated the absolute values of the correlation weights for each descriptor. This is because the Random Forest standard variable importance measures do not take account of the sign of the contribution a given descriptor value makes towards the prediction. 
274 The Random Forest algorithm implemented in the randomForest R package which was used in this work 275 provided information on variable importance using two approaches, by setting the "importance" option of the randomForest function to TRUE. The first method [25] calculates the percentage increase of the mean squared error ("\%IncMSE") on the out-of-bag (OOB) subset - i.e. the subset of training set instances not used to build a given tree - after the permutation of descriptors' values. In greater detail, for each tree in the forest, the prediction error on the OOB portion of the data, expressed by the mean square error (MSE) is recorded (for regression problems). The MSE value is then calculated again after permuting each predictor variable one at a time. The differences between the two calculated MSEs for the original and shuffled datasets are averaged over all trees and then normalised by the standard deviation of the differences. The second method ("IncNodePurity") calculates the total decrease in node “impurities" from splitting on a given descriptor, averaged over all the generated trees. For regression, "impurity" is measured by the residual sum of squares (RSS) metric for a given node [27].

\subsection{Summarising Variable Importance Values}

The different variable importance approaches employed with Random Forest and CORAL are applicable for a single model, hence - in order to derive general conclusions - it was necessary to summarise these, for a given combination of modelling approach and variable importance approach, over all 95 (19 LOO training sets $\times 5$ repetitions) models. Furthermore, it was necessary to take account of the fact that the different approaches could vary in scale - which would confound comparisons. Hence, the raw values (v) - for a given combination of modelling approach and variable importance approach - were scaled ( $\left.\mathrm{v}_{\text {scaled }}\right)$ between 0 and 1 as per equation (8), where the minimum $\left(\mathrm{v}_{\min }\right)$ and maximum $\left(\mathrm{v}_{\max }\right)$ values were obtained across all 95 models and all 5 descriptors. Subsequently, the values were summarised in terms of the arithmetic mean and the corresponding standard error of the mean.

297

$\mathbf{v}_{\text {scaled }}=\frac{\left(\mathbf{v}-\mathbf{v}_{\min }\right)}{\left(\mathbf{v}_{\max }-\mathbf{v}_{\min }\right)}$

\subsection{Feature contribution analysis}

By "feature contribution analysis", we refer to estimates of both the sign and magnitude of the influence 
measures which only estimate the magnitude of the influence. As far as the CORAL software is concerned, we calculated feature contributions based on the signed values of the correlation weights. Indeed, as equations (6) and (7) show (see the "Variable importance" section), for each single model which is obtained by selecting the additive method, the signed values of the correlation weights allow understanding of whether a certain descriptor is contributing "positively", i.e. it contributes to increased toxicity, or "negatively", i.e. it contributes to decreased toxicity. For Random Forest, a feature contribution analysis was carried out using the technique developed by Kuz'min and colleagues [45] and implemented in the rfFC $\mathrm{R}$ package [44] which is designed to work with the randomForest implementation of Random Forest. (Specifically, version 1.0 of $\mathrm{rfFC}$, as obtained via the “install.packages("rfFC",repos="http://R-Forge.R-project.org")" command, was used in the current work). This feature contribution method is a measure of the influence, in terms of the magnitude and sign, of each variable on the model prediction for a single instance. In principle, the feature contribution associated with the value of a given descriptor could vary between instances with the same value for that given descriptor, due to the fact that Random Forest models are non-linear. In contrast, the feature contribution associated with a single descriptor as calculated for CORAL is either equal to the value of the corresponding correlation weight (if the descriptor value is 1 ) or 0 (if the descriptor value is 0 ). Hence, to enable a comparison between the average influence of a given descriptor value being 1 for both CORAL and Random Forest, pseudo-coefficients were derived from the Random Forest feature contributions. These pseudo-coefficients were calculated by computing, for each descriptor, the difference between the arithmetic mean average values calculated over the feature contribution values for the pseudo-SMILES strings having a value of 1 for that specific descriptor (here called FC(1)) and pseudo-SMILES strings having a value of 0 for the same descriptor (here called $\mathrm{FC}(0)$ ), according to the equation (9). LOO results, in terms of $\mathrm{R}^{2}$ and RMSE for both CORAL and Random Forest algorithms are reported in Table 4. As far as the global results on the corresponding training sets are concerned, the average and standard error of the mean, over the 95 developed models, of the $\mathrm{R}^{2}$ and RMSE statistics were calculated for both CORAL and Random Forest models. N.B. In contrast to the results shown in Table 4, results 
with perfectly correlated descriptors (for both CORAL and Random Forest) and bootstrap sampling (for

334 Random Forest) are presented in the SI. Considering the LOO results reported in Table 4, it is clear that CORAL's LOO test set performance was substantially worse than its performance on the corresponding training sets. With respect to CORAL, the global average value of the $\mathrm{R}^{2}$ on training sets was 0.8285 whereas the average RMSE was 0.2347. Results from LOO (i.e. testing) for CORAL showed a decrease for the average $\mathrm{R}^{2}$ to 0.6486 , whereas the average value of the RMSE increased to 0.3456 . However, the corresponding results for Random Forest showed a smaller reduction in estimated model performance upon going between training and LOO test global results. The average values of $\mathrm{R}^{2}$ were 0.8723 and 0.7807 for training and test set respectively and the average values for RMSE were 0.2011 and 0.2604 for training and test set respectively. If one considers only results from the LOO test sets in Table 4, it can be stated that Random Forest performed better that CORAL and the smaller reduction in average model performance upon going from the training to the test sets indicates Random Forest did not overfit as much. As far as single run results are concerned, as shown in Table 4 for CORAL software, the average $\mathrm{R}^{2}$ values on LOO training sets, for different splits of the same LOO training sets to yield internal "test sets" for hyperparameter selection, ranged between 0.7876 and 0.8570 . (Here, it should be remembered that - for a given split of the data to yield internal "tests sets" for each LOO training set - the results were averaged across all 19 LOO training sets.) Corresponding LOO $\mathrm{R}^{2}$ test set values ranged between 0.6143 and 0.7082. Average RMSE values for different splits of the CORAL input dataset ranged from 0.2119 and 0.2675 on training sets whereas RMSE values on test sets ranged between 0.3010 and 0.3712 . The Random Forest approach showed less variability, in terms of both $\mathrm{R}^{2}$ and RMSE, among the five runs of the software with different seeds. Indeed, average training set $\mathrm{R}^{2}$ values ranged between 0.8711 and 0.8736, whereas LOO test set $\mathrm{R}^{2}$ values ranged between 0.7707 and 0.7899 . Moreover, according to Table 4, Random Forest average RMSE values ranged between 0.1995 and 0.2022 on training sets whereas on LOO test sets RMSE values ranged between 0.2544 and 0.2665 . It is important to note that, among the five runs of $\mathrm{LOO}$ for the CORAL software, the largest difference in the $\mathrm{R}^{2}$ values between training and test sets is 0.2427 (split 3) whereas, for Random Forest, the largest difference is 0.1004. Taking into account the reference value for the difference of $\mathrm{R}^{2}$ between training and test sets reported in the article of Eriksson and colleagues [46], the average results obtained with CORAL are closer than Random Forest to the 0.3 threshold for which a model could be considered to overfit. Additional results obtained with CORAL and Random Forest under different scenarios are presented in Table S1 in the SI. Firstly, it can 
be observed that no significant training/test set performance gap exists for Random Forest if the training set is predicted using only out-of-bag samples. Furthermore, the comparison of the results obtained with and without correlated descriptors for the dataset used in this work showed that Random Forest is, as expected [25], less affected than CORAL by the presence of correlated descriptors (see Table S1 in SI). Specifically, the split number 2 of the CORAL input dataset generated an outlier only when perfectly correlated descriptors were used. For Random Forest, a very small difference in terms of $\mathrm{R}^{2}$ and RMSE global average values was observed for results with and without bootstrap sampling.

\subsection{Variable importance results}

Figure 2 shows the average and standard error of the mean (as error bars) of the scaled variable importance values for each descriptor and each variable importance measure for CORAL and Random Forest. N.B. In contrast to the results shown in Figure 2, results with perfectly correlated descriptors (for both CORAL and Random Forest) and bootstrap sampling (for Random Forest) are presented in the SI (Figures S1 and $\mathrm{S} 2$ ). As far as CORAL is concerned, the average values ranged between 0.0525 and 0.8941 for the $\mathrm{K}$ and L descriptors, respectively, which are related to the nanoparticle aspect ratio (i.e. aspect ratio > 1) and zeta potential (zeta potential $\geq-32.0 \mathrm{mV}$ ) nanomaterial physico-chemical properties respectively. Hence, according to the CORAL variable importance measure, the nanoparticle aspect ratio and zeta potential were respectively the least and most important variables related to cytotoxicity. With respect to the Random Forest \%IncMSE method, the average values ranged between 0.0424 and 0.8393 for the G and L descriptors which are related to the THP-1 cell line and zeta potential respectively. On the other hand, Random Forest IncNodePurity method average values ranged between 0.0124 and 0.8181 for the E and L descriptors which refer to the HaCaT cell line and zeta potential respectively. In spite of small differences depending upon the specific method used, the descriptors ( $\mathrm{K}$ and $\mathrm{L}$ ) corresponding to aspect ratio and zeta potential are (on average) by far the most important according to both the Random Forest variable importance measures. Conversely, even if CORAL also identified the descriptor corresponding to zeta potential as the most important, descriptors D and G corresponding to cell lines A549 and THP-1, respectively are the second and third most important variables. Furthermore, Random Forest variable importance results with perfectly correlated descriptors also support the conclusion that aspect ratio and zeta potential are the most toxicologically relevant variables, confirming that the Random Forest approach is not significantly affected by correlated descriptors (see Figure S2 in SI). Similarly, Random Forest 
variable importance results obtained without bootstrap sampling were largely consistent with those obtained with bootstrap sampling, for both \%IncMSE and IncNodePurity methods (see Figure S1 and Figure S2 in SI). CORAL variable importance results with correlated descriptors showed that the two most important variables were the $\mathrm{J}$ and $\mathrm{L}$ descriptors, corresponding to the aspect ratio and zeta potential. However, it is important to note that, unlike for Random Forest, the other descriptors corresponding to aspect ratio and zeta potential are not similarly important. It is also important to note that, for CORAL, the variable importance values calculated with or without perfectly correlated descriptors were not as consistent as compared to Random Forest.

\subsection{Feature contribution results}

Figure 3 shows the average values, for both CORAL correlation weights and Random Forest feature contribution pseudo-coefficients, calculated across all the 95 models generated on the LOO training sets, without perfectly correlated descriptors and without bootstrap sampling for Random Forest. (Results with perfectly correlated descriptors are presented in SI Figure S3.) Broadly in keeping with what was observed for the variable importance analysis (Figure 2), for Random Forest aspect ratio and zeta potential nanoparticles' physico-chemical properties were the two most important variables whereas, for the CORAL approach, zeta potential and the variable related to the A549 cell line appear most important. Hence, as expected, feature contribution results are consistent with those obtained for variable importance for both approaches. It is important to note that, for CORAL approach, feature contribution average values were all positive. Conversely, Random Forest feature contribution results presented both positive and negative values. Specifically, for the CORAL approach, the correlation weight associated with the zeta potential feature had a magnitude that is more than double of the A549 cell line magnitude; whereas for Random Forest the two highest feature contribution values have a similar magnitude. In addition, in contrast to CORAL, for Random Forest there is a considerable difference between the average influence of the two most important descriptors (relating to aspect ratio and zeta potential) and the others. These observations regarding the importance of different variables according to the feature contributions calculations (Figure 3) are broadly in keeping with those observed when perfectly correlated descriptors are not excluded (Figure S3). Results with correlated descriptors in the SI (Figure S3) showed that once again for Random Forest approach zeta potential and aspect ratio were the two most important properties. Specifically, considering the two correlated descriptors for aspect ratio and zeta potential, namely the $\mathbf{J}$ and $\mathrm{K}$ labels for aspect ratio and the $\mathrm{L}$ and $\mathrm{M}$ labels for zeta potential, it is worth noting that, for Random 
423 Forest, the magnitudes of their average feature contribution values were not only very similar (roughly

424 0.23) but also much greater than the magnitudes for the other descriptors. Conversely, for CORAL, we 425 obtained average feature contributions of significantly different magnitude for the two correlated 426 descriptors related to the same variable, both for aspect ratio and zeta potential properties. When the 427 signed values are considered (Figure 3 or Figure S3), it is worth noting that for Random Forest high values of zeta potential are associated with an increase in cytotoxicity, whereas high aspect ratio values are associated with a decrease of toxicity since the average pseudo-coefficient value is negative for the corresponding descriptors. It is important to note that these findings are consistent with the preliminary analysis of the dataset reported in Figure 1. Conversely, the CORAL approach seems to only be able to partially recognise the trend in the data for the zeta potential. The descriptor associated with higher zeta potential values has a positive average feature contribution value, regardless of whether perfectly correlated descriptors were removed (Figure 3) or not (Figure S3). However, when perfectly correlated descriptors are not removed, the average feature contribution value for the descriptor corresponding to lower zeta potential values is still positive, even if less so (Figure S3). Whether perfectly correlated descriptors were removed (Figure 3) or not (Figure S3), the average feature contribution value for both descriptors corresponding to aspect ratio was positive.

\section{Discussion}

440 Taking into account the results obtained in this comparison work, both in terms of their predictive performance estimated via "external" LOO validation and their ability to be interpreted to reveal trends in the data, the non-linear Random Forest approach performed better than the linear CORAL approach for the specific dataset used in this paper. With respect to Random Forest, the difference for both $\mathrm{R}^{2}$ and RMSE average values between training and test sets was smaller and it had better results on the test set compared to CORAL (Table 4). In addition, for Random Forest both average $\mathrm{R}^{2}$ and RMSE values for the OOB and LOO predictions methods were very similar, regardless of whether modelling was performed with or without bootstrap sampling and with or without correlated descriptors (Table S1). This is interesting since it suggests that, even for these small datasets, as is typical for nano-QSAR studies [47], there may be no need to cross-validate Random Forest models as opposed to simply reporting their OOB performance. (Of course, for comparing to other methods, cross-validation would still be required for a fair, like-for-like comparison). However, it must be noted that this finding may not hold in general, e.g. Ballester and Mitchell found the OOB predictions only converged to the test set performance as the 
training set got larger [48]. Currently, there is an on-going discussion on the importance of so-called

454 intrinsic and extrinsic properties as well as composition of nanoparticles for toxicological studies [10, 13,

455 49]. In our work, we incorporated various intrinsic (e.g. average primary particle size) and extrinsic (e.g.

456 zeta potential) properties as descriptors for the modelled toxicity endpoint. We further sought to take

457 account of variability in the endpoint values due to the different experimental conditions, by treating the

458 varied experimental conditions as additional descriptors, as per the so-called "eclectic" approach

459 previously proposed in the literature $[41,42,50]$. The variable importance analysis performed in this work

460 showed that the aspect ratio and zeta potential nanoparticles' physico-chemical properties were the most

461 important variables for the Random Forest approach under all modelling scenarios with or without

462 perfectly correlated descriptors and with or without bootstrap sampling (Figure 2, Figure S1 and Figure

463 S2). This was not observed for CORAL. For example, when modelling was carried out without perfectly

464 correlate descriptors (Figure 2), the two most important descriptors related to zeta potential and the A549

465 cell line. In contrast to the results obtained with Random Forest, for which the most important descriptors

466 - associated with zeta potential and aspect ratio - were comparably important, zeta potential was more

467 important for CORAL than the A549 cell line, which had a comparable importance to the THP-1 cell line

468 (Figure 2). However, it must be noted that descriptors related to cell line appear relatively less important

469 when perfectly correlate descriptors are not removed from CORAL modelling (Figure S2). Regarding the

470 observations concerning the importance of descriptors related to cell lines, Kim and colleagues [51]

471 recently reported that cell type more than other factors like nanoparticles' size and dose level can influence

472 cytotoxicity and, in addition, in the same work they stated that identical nanoparticles' preparations yield

473 different outcomes depending on the selected cell lines even if they belong to the same cell type. Whilst

474 our findings are not directly comparable, they still suggest that cell line is at least as important an

475 experimental variable as average size, with the exact significance varying depending upon the specific

476 cell line, the specific variable importance approach and modelling scenario (Figure 2, Figure S1 and

477 Figure S2). As far as nanoparticles' size is concerned, the work of Rong and colleagues [52] showed a

478 potential important role of silica particles' sizes in increasing toxicity towards endothelial cells. In another

479 more relevant study, Tokgun and colleagues [53] reported results which showed that cytotoxicity towards

480 A549 cell line depends on silica nanoparticles' size. We found that the average size of silica nanoparticles

481 was not typically (Figure 2, Figure S1 and Figure S2) amongst the most important variables but it did

482 appear more significant when CORAL modelling was carried out including perfectly correlated 

between cytotoxicity and both zeta potential and aspect ratio (Figure 1) which was also reflected in the Random Forest variable importance (Figure 2, Figure S1 and Figure S2) and feature contributions (Figure 3 and Figure S3) calculations. The clear correspondence between both the average Random Forest variable importance and feature contributions and the clear trends observed in the dataset makes it clear that our findings are not a result of an artefact of modelling but rather a consequence of the experimental dataset used in this work. However, these clear trends observed in the dataset appear to be at odds with the literature. Various publications have previously considered the relationship between aspect ratio and zeta potential nanoparticles' physico-chemical properties and cytotoxicity. Regarding the toxicological significance of particle shape (as quantified via the aspect ratio), studies for both carbon nanotubes and silica nanoparticles (as per the current work) either reported that aspect ratio had no relationship to toxicity or that high aspect ratio particles are more toxic $[54,55]$. In contrast, if we look at the specific dataset used in this work, as shown in Figure 1, high aspect ratio silica nanoparticles are clustered at the low toxicity side of the graph. This finding is also reflected in the average Random Forest pseudo-coefficients presented in Figure 3. This discrepancy may be due to several reasons, such as differences in other characteristics of nanomaterials or in the cytotoxicity protocol used or in the cell line adopted as well as the concentrations selected for the test. To this respect, the review of Fruijtier-Pölloth and colleagues [56] has shown that it is difficult to compare studies that are based on different experimental conditions and nanomaterials since they could yield contradictory results, which might be due to diverse toxicological mechanisms involved. As far as the relationship between zeta potential and toxicity is concerned, Cho et al. [57] found that, for a set of metal/metal oxide/silica nanoparticles high positive zeta potential resulted in more cytotoxicity and Karunakaran et al. [58] also suggested that cytotoxicity of alumina and silica particles, both micro-sized and nanoparticles, increases as a result of positive zeta potential. In the current work, both the feature contribution analysis results, as shown in Figure 3, and the preliminary analysis of the data shown in Figure 1, revealed that less negative zeta potential values were associated with higher cytotoxicity and that this trend was clearly captured by Random Forest and, to a lesser extent, CORAL (see Figure 3 and Figure S3). Whilst this might be considered consistent with earlier indications that increasing zeta potential leads to higher cytotoxicity [57, 58], it must be stressed that these earlier studies indicated that it was specifically positive zeta potential values that led to higher cytotoxicity and all zeta potential values reported in the dataset used for the current work were negative. One possible confounding 
513 factor here could be that zeta potential is highly dependent upon the composition of the medium in which

514 it was measured [57] and the experimentalists who provided the data modelled in the current work

515 indicated that zeta potential values were measured in water rather than the exposure medium used for

516 cytotoxicity testing. Hence, the actual zeta potential values of the nanoparticles when they were exposed

517 to the cells could differ from those reported in our dataset. Arguably, better mechanistic insight would be

518 obtained if zeta potential values had been measured under biologically relevant conditions [49, 57]. It is

519 also the case that future studies might build upon our work via incorporating additional descriptors into

520 the models. Firstly, as shown in the electronic version of the dataset used in this work in the SI the original

521 dataset from which this was derived included other nanomaterial characteristics and experimental

522 variables that were not considered as descriptors in our current work e.g. serum concentration or

523 dispersion protocol. Indeed, prior to modelling analyses, we selected only five variables to model,

524 according to our expert judgement since serum concentration and, supposing stirring and vortexing

525 protocols were comparable, dispersion protocol experimental values, for this specific dataset, were the

526 same for 17 out of 19 instances of the original dataset. (The assumption that the stirring and vortexing

527 protocols were comparable was based on guidance from the MODENA COST team responsible for this

528 dataset.) Secondly, none of the parameters provided in the original dataset may be considered to capture

529 the surface reactivity or dissolution of the studied silica nanoparticles. One way of partially addressing

530 this in future work, other than making additional experimental measurements [13], would be to perform

531 additional quantum-mechanical calculations to obtain new different descriptors, i.e. independent variables

532 reflecting structural and chemical properties of the nanoparticles [14, 59]. Such variables could further

533 enhance our understanding of the possible mechanism of toxicity of the studied nanoparticles.

\section{5. Conclusions}

535 In this work a comparison between the CORAL and Random Forest methods in predicting silica 536 nanoparticles' cytotoxicity, based upon physico-chemical characteristics and experimental conditions 537 encoded into pseudo-SMILES strings, was performed. It was demonstrated that the pseudo-SMILES 538 encoding proposed for CORAL could be translated into descriptors which can be used with other 539 modelling approaches, such as Random Forest. LOO was used to externally validate the results obtained 540 from the modelling task. The predictive performance estimated from LOO was significantly higher with 541 Random Forest and substantially less overfitting was observed. Different approaches were employed to 
analyse the significance of different descriptors within both kinds of models, including the derivation of pseudo-coefficients for Random Forest models that, in contrast to standard variable importance measures, reflect the signed contribution of descriptors towards the modelled endpoint. Whilst differences were observed with the different approaches to interpreting the models, the Random Forest approach, more than CORAL, reflected the toxicological significance of zeta potential and aspect ratio observed from preliminary analysis of the dataset. Interestingly, whilst these properties have previously been reported as significant for nanomaterial toxic effects, the relationships observed here were not in complete agreement with some previous studies - which could reflect different mechanisms. In summary, the results obtained suggest the Random Forest modelling approach is readily applicable to modelling the cytotoxicity of nanoparticles and can be used to develop models which offer reasonable predictive power and which can be interpreted in terms of physico-chemical-toxicity relationships.

\section{Acknowledgements}

MC and RLMR are grateful for funding from the European Union Seventh Framework Programme (FP7/2007-2013) under grant agreement number 309837 (NanoPUZZLES project). TP and AG are grateful for funding from the Polish Ministry of Science and Higher Education under grant agreement DS 530-8637-D510-15. The MODENA COST Initiative (Grant Information - COST TD1204 'MODENA') and its experimental partners are thanked for providing the experimental data used in this work. The authors also thank Dr. Andrey A. Toropov and Dr. Alla P. Toropova of the Mario Negri Institute for Pharmacological Research (Italy) for their support in the use of the CORAL software.

\section{References}

1. Lövestam, G., Rauscher, H., Roebben, G., Klüttgen, B. S., Gibson, N., Putaud, J.-P., \& Stamm, H. (2010). Considerations on a definition of nanomaterial for regulatory purposes: Publications Office.

2. Bolt, H., Marchan, R., \& Hengstler, J. (2013). Recent developments in nanotoxicology. Archives of toxicology, 1-2.

3. Kumar, A., \& Dhawan, A. (2013). Genotoxic and carcinogenic potential of engineered nanoparticles: an update. Archives of toxicology, 87(11), 1883-1900.

4. Gebel, T., Foth, H., Damm, G., Freyberger, A., Kramer, P.-J., Lilienblum, W., Röhl, C., Schupp, T., Weiss, C., Wollin, K.-M., Hengstler, J.G. (2014). Manufactured nanomaterials: categorization and approaches to hazard assessment. Archives of toxicology, 88(12), 2191-2211.

5. Huo, L., Chen, R., Shi, X., Bai, R., Wang, P., Chang, Y., \& Chen, C. (2015). High-Content Screening for Assessing Nanomaterial Toxicity. Journal of nanoscience and nanotechnology, 15(2), 1143-1149. 
6. Muthuraman, P., Ramkumar, K., \& Kim, D. H. (2014). Analysis of dose-dependent effect of zinc oxide nanoparticles on the oxidative stress and antioxidant enzyme activity in adipocytes. Applied biochemistry and biotechnology, 174(8), 2851-2863.

7. Sre, P. R., Reka, M., Poovazhagi, R., Kumar, M. A., \& Murugesan, K. (2015). Antibacterial and cytotoxic effect of biologically synthesized silver nanoparticles using aqueous root extract of Erythrina indica lam. Spectrochimica Acta Part A: Molecular and Biomolecular Spectroscopy, 135, 1137-1144.

8. El Mahdy, M. M., Eldin, T. A. S., Aly, H. S., Mohammed, F. F., \& Shaalan, M. I. (2015). Evaluation of hepatotoxic and genotoxic potential of silver nanoparticles in albino rats. Experimental and Toxicologic Pathology, 67(1), 21-29.

9. Donaldson, K., \& Poland, C. A. (2013). Nanotoxicity: challenging the myth of nano-specific toxicity. Current opinion in biotechnology, 24(4), 724-734.

10. Lynch, I., Weiss, C., \& Valsami-Jones, E. (2014). A strategy for grouping of nanomaterials based on key physico-chemical descriptors as a basis for safer-by-design NMs. Nano Today, 9(3), 266-270.

11. OECD. (2014). Guidance on grouping of chemicals. Series on testing and assessment No. 194 (second ed.).

12. Miller, J. B., \& Hobbie, E. K. (2013). Nanoparticles as macromolecules. Journal of Polymer Science Part B: Polymer Physics, 51(16), 1195-1208.

13. Stefaniak, A. B., Hackley, V. A., Roebben, G., Ehara, K., Hankin, S., Postek, M. T., Lynch, I., Fu, W.-E., Linsinger, T. P. J., \& Thünemann, A. F. (2013). Nanoscale reference materials for environmental, health and safety measurements: needs, gaps and opportunities. Nanotoxicology, 7(8), 1325-1337.

14. Puzyn, T., Rasulev, B., Gajewicz, A., Hu, X., Dasari, T. P., Michalkova, A., Hwang, H.-M., Toropov, A. A., Leszczynska, D., \& Leszczynski, J. (2011). Using nano-QSAR to predict the cytotoxicity of metal oxide nanoparticles. Nature nanotechnology, 6(3), 175-178.

15. Ying, J., Zhang, T., \& Tang, M. (2015). Metal Oxide Nanomaterial QNAR Models: Available Structural Descriptors and Understanding of Toxicity Mechanisms. Nanomaterials, 5(4), 1620-1637.

16. Winkler, D. A. (2015). Recent advances, and unresolved issues, in the application of computational modelling to the prediction of the biological effects of nanomaterials. Toxicology and applied pharmacology.

17. Gramatica, P., Chirico, N., Papa, E., Cassani, S., \& Kovarich, S. (2013). QSARINS: A new software for the development, analysis, and validation of QSAR MLR models. Journal of Computational Chemistry, 34(24), 2121-2132.

18. Bigdeli, A., Hormozi-Nezhad, M. R., \& Parastar, H. (2015). Using nano-QSAR to determine the most responsible factor (s) in gold nanoparticle exocytosis. RSC Advances, 5(70), 57030-57037.

19. Müller, K.-R., Mika, S., Rätsch, G., Tsuda, K., \& Schölkopf, B. (2001). An introduction to kernelbased learning algorithms. Neural Networks, IEEE Transactions on, 12(2), 181-201.

20. Hsu, C.-W., Chang, C.-C., \& Lin, C.-J. (2003). A practical guide to support vector classification.

21. Panaye, A., Fan, B., Doucet, J., Yao, X., Zhang, R., Liu, M., \& Hu, Z. (2006). Quantitative structuretoxicity relationships (QSTRs): A comparative study of various non linear methods. General regression neural network, radial basis function neural network and support vector machine in predicting toxicity of nitro-and cyano-aromatics to Tetrahymena pyriformis §. SAR and QSAR in Environmental Research, 17(1), 75-91.

22. Liu, R., Rallo, R., Weissleder, R., Tassa, C., Shaw, S., \& Cohen, Y. (2013). Nano-SAR development for bioactivity of nanoparticles with considerations of decision boundaries. Small, 9(9-10), 1842-1852.

23. Liu, R., Rallo, R., Bilal, M., \& Cohen, Y. (2015). Quantitative structure-activity relationships for cellular uptake of surface-modified nanoparticles. Combinatorial chemistry \& high throughput screening, 18(4), 365-375.

24. Breiman, L. (2001). Random forests. Machine learning, 45(1), 5-32. 
25. Svetnik, V., Liaw, A., Tong, C., Culberson, J. C., Sheridan, R. P., \& Feuston, B. P. (2003). Random forest: a classification and regression tool for compound classification and QSAR modeling. Journal of chemical information and computer sciences, 43(6), 1947-1958.

26. Toropov, A. A., Toropova, A. P., Mukhamedzhanova, D. V., \& Gutman, I. (2005). Simplified molecular input line entry system (SMILES) as an alternative for constructing quantitative structureproperty relationships (QSPR). INDIAN JOURNAL OF CHEMISTRY SECTION A, 44(8), 1545.

27. Liaw, A., \& Wiener, M. (2002). Classification and regression by randomForest. R news, 2(3), 18-22.

28. Sizochenko, N., Jagiello, K., Leszczynski, J., \& Puzyn, T. (2015). How the "Liquid Drop" Approach Could Be Efficiently Applied for Quantitative Structure-Property Relationship Modeling of Nanofluids. The Journal of Physical Chemistry C, 119(45), 25542-25547.

29. Goldberg, E., Scheringer, M., Bucheli, T. D., \& Hungerbühler, K. (2015). Prediction of nanoparticle transport behavior from physicochemical properties: machine learning provides insights to guide the next generation of transport models. Environmental Science: Nano, 2(4), 352-360.

30. Ngamwongsatit, P., Banada, P. P., Panbangred, W., \& Bhunia, A. K. (2008). WST-1-based cell cytotoxicity assay as a substitute for MTT-based assay for rapid detection of toxigenic Bacillus species using CHO cell line. Journal of Microbiological Methods, 73(3), 211-215.

31. Mosmann, T. (1983). Rapid colorimetric assay for cellular growth and survival: application to proliferation and cytotoxicity assays. Journal of immunological methods, 65(1-2), 55-63.

32. Domey, J., Haslauer, L., Grau, I., Strobel, C., Kettering, M., \& Hilger, I. (2013). Probing the cytotoxicity of nanoparticles: experimental pitfalls and artifacts.

33. OECD. (2012). Guidance on sample preparation and dosimetry for the safety testing on manufactured nanomaterials (version of 20 June 2012).

34. Martin, J. K., \& Hirschberg, D. S. (1996). Small sample statistics for classification error rates I: Error rate measurements: Information and Computer Science, University of California, Irvine.

35. Hawkins, D. M., Basak, S. C., \& Mills, D. (2003). Assessing model fit by cross-validation. Journal of chemical information and computer sciences, 43(2), 579-586.

36. Alexander, D., Tropsha, A., \& Winkler, D. A. (2015). Beware of R 2: Simple, Unambiguous Assessment of the Prediction Accuracy of QSAR and QSPR Models. Journal of chemical information and modeling, 55(7), 1316-1322.

37. Hawkins, D. M. (2004). The problem of overfitting. Journal of chemical information and computer sciences, 44(1), 1-12.

38. Low, Y., Uehara, T., Minowa, Y., Yamada, H., Ohno, Y., Urushidani, T., Alexander Sedykh, A., Muratov, E., Kuz'min, V., Fourches, D., Zhu, H., Rusyn, I., \& Tropsha, A. (2011). Predicting druginduced hepatotoxicity using QSAR and toxicogenomics approaches. Chemical research in toxicology, 24(8), 1251-1262.

39. Marchese Robinson, R. L., Glen, R. C., \& Mitchell, J. B. O. (2011). Development and comparison of hERG blocker classifiers: Assessment on different datasets yields markedly different results. Molecular Informatics, 30(5), 443-458.

40. Manganelli, S., Leone, C., manganelli, A. A., Toropova, A. P., \& Benfenati, E. (2016). QSAR model for predicting cell viability of human embryonic kidney cells exposed to $\mathrm{SiO} 2$ nanoparticles. Chemosphere, 144, 995-1001.

41. Toropov, A. A., Toropova, A. P., Veselinovic, A. M., Veselinovic, J. B., Nesmerak, K., Raska, J., Duchowicz, P. A., Castro, E. O., Kudyshkin, V., Leszczynska, D., Leszczynski, J., Leszczynska, D. (2015). The Monte Carlo Method Based on Eclectic Data as an Efficient Tool for Predictions of Endpoints for Nanomaterials-Two Examples of Application. Combinatorial chemistry \& high throughput screening, 18(4), 376-386. 
42. Toropov, A. A., \& Toropova, A. P. (2014). Optimal descriptor as a translator of eclectic data into endpoint prediction: Mutagenicity of fullerene as a mathematical function of conditions. Chemosphere, $104,262-264$.

43. Toropov, A. A., Toropova, A. P., Benfenati, E., Gini, G., Puzyn, T., Leszczynska, D., \& Leszczynski, J. (2012). Novel application of the CORAL software to model cytotoxicity of metal oxide nanoparticles to bacteria Escherichia coli. Chemosphere, 89(9), 1098-1102.

44. Palczewska, A., Palczewski, J., Marchese Robinson, R. L., \& Neagu, D. (2014). Interpreting random forest classification models using a feature contribution method Integration of Reusable Systems (pp. 193-218): Springer.

45. Kuz'min, V. E., Polishchuk, P. G., Artemenko, A. G., \& Andronati, S. A. (2011). Interpretation of QSAR models based on random forest methods. Molecular Informatics, 30(6-7), 593-603.

46. Eriksson, L., Jaworska, J., Worth, A. P., Cronin, M. T., McDowell, R. M., \& Gramatica, P. (2003). Methods for reliability and uncertainty assessment and for applicability evaluations of classification-and regression-based QSARs. Environmental health perspectives, 111(10), 1361.

47. Oksel, C., Ma, C., \& Wang, X. (2015). Current situation on the availability of nanostructurebiological activity data. SAR and QSAR in Environmental Research, 26(2), 79-94.

48. Ballester, P. J., \& Mitchell, J. B. (2010). A machine learning approach to predicting protein-ligand binding affinity with applications to molecular docking. Bioinformatics, 26(9), 1169-1175.

49. Marchese Robinson, R. L., Lynch, I., Peijnenburg, W., Rumble, J., Klaessig, F., Marquardt, C., Rauscher, H., Puzyn, T., Purian, R., Åberg, C., Karcher, S., Vriens, H., Hoet, P., Hoover, M. D., Hendren, C. O., \& Harper., S. L. (2016). How should the completeness and quality of curated nanomaterial data be evaluated? Nanoscale.

50. Toropova, A. P., Toropov, A. A., Manganelli, S., Leone, C., Baderna, D., Benfenati, E., \& Fanelli, R. (2016). Quasi-SMILES as a tool to utilize eclectic data for predicting the behavior of nanomaterials. NanoImpact.

51. Kim, I.-Y., Joachim, E., Choi, H., \& Kim, K. (2015). Toxicity of silica nanoparticles depends on size, dose, and cell type. Nanomedicine: Nanotechnology, Biology and Medicine, 11(6), 1407-1416.

52. Rong, Y., Zhou, T., Cheng, W., Guo, J., Cui, X., Liu, Y., \& Chen, W. (2013). Particle-size-dependent cytokine responses and cell damage induced by silica particles and macrophages-derived mediators in endothelial cell. Environmental toxicology and pharmacology, 36(3), 921-928.

53. Tokgun, O., Demiray, A., Kaya, B., Karagür, E. R., Demir, E., Burunkaya, E., \& Akça, H. (2015). SILICA NANOPARTICLES CAN INDUCE APOPTOSIS VIA DEAD RECEPTOR AND CASPASE 8 PATHWAY ON A549 CELLS.

54. Donaldson, K., Murphy, F. A., Duffin, R., \& Poland, C. A. (2010). Asbestos, carbon nanotubes and the pleural mesothelium: a review of the hypothesis regarding the role of long fibre retention in the parietal pleura, inflammation and mesothelioma. Particle and fibre toxicology, 7(1), 1.

55. Yu, T., Malugin, A., \& Ghandehari, H. (2011). Impact of silica nanoparticle design on cellular toxicity and hemolytic activity. ACS nano, 5(7), 5717-5728.

56. Fruijtier-Pölloth, C. (2012). The toxicological mode of action and the safety of synthetic amorphous silica-A nanostructured material. Toxicology, 294(2), 61-79.

57. Cho, W.-S., Duffin, R., Thielbeer, F., Bradley, M., Megson, I. L., MacNee, W., Poland, C. A., Tran, L., \& Donaldson, K. (2012). Zeta potential and solubility to toxic ions as mechanisms of lung inflammation caused by metal/metal-oxide nanoparticles. Toxicological Sciences.

58. Karunakaran, G., Suriyaprabha, R., Rajendran, V., \& Kannan, N. (2015). Effect of contact angle, zeta potential and particles size on the in vitro studies of $\mathrm{Al} 2 \mathrm{O} 3$ and $\mathrm{SiO} 2$ nanoparticles. Nanobiotechnology, IET, 9(1), 27-34. 
716

717

718

719

720

721

722

723

724

725

726

727

728

729

730

731

732

733

734

735

736

737

738

739

740

741

742

743

744

745

746

747

748

749
59. Gajewicz, A., Schaeublin, N., Rasulev, B., Hussain, S., Leszczynska, D., Puzyn, T., \& Leszczynski, J. (2015). Towards understanding mechanisms governing cytotoxicity of metal oxides nanoparticles: Hints from nano-QSAR studies. Nanotoxicology, 9(3), 313-325.

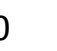

22

23

5

6

4

35

7

38

40




\section{Tables}

751 Table 1: In vitro WST-1 cytotoxicity experimental data of silica nanomaterials used for modelling.

752 "Treatment time" and "Cell type" columns are related to the in vitro experimental conditions adopted

753 during the experimental test for the exposure duration and cell line model, respectively. "Average size",

754 "Aspect ratio" and "Zeta potential" columns are related to measured physico-chemical properties for size,

755 aspect ratio and zeta potential of each nanomaterial, respectively. The average size was calculated from

756 two primary size dimensions estimated by TEM or other measurements (see the ESI for more details).

757 The "pEC25" column is the modelled variable, namely the negative logarithm, to base 10, of the EC25

758 value expressed as surface area of nanomaterial per millilitre (i.e. $\mathrm{mm} 2 / \mathrm{ml}$ ). Units are reported in squared

759 brackets for numerical properties.

\begin{tabular}{|c|c|c|c|c|c|c|}
\hline ID & $\begin{array}{c}\text { Treatment } \\
\text { time }[\mathrm{h}]\end{array}$ & Cell type & $\begin{array}{c}\text { Average } \\
\text { size } \\
{[\mathrm{nm}]}\end{array}$ & $\begin{array}{c}\text { Aspect ratio } \\
\text { [adimensional] }\end{array}$ & $\begin{array}{c}\text { Zeta } \\
\text { potential } \\
{[\mathrm{mV}]}\end{array}$ & $\begin{array}{c}\mathrm{pEC}_{25} \\
{\left[\mathrm{~mm}^{2} / \mathrm{ml}\right]}\end{array}$ \\
\hline 119 & 24 & THP-1 & 20.0 & 1.4 & -46.1 & -1.299 \\
\hline 104 & 24 & $16 \mathrm{HBE}$ & 46.0 & 1.2 & -40.0 & -1.272 \\
\hline 186 & 48 & THP-1 & 18.0 & 1.6 & -43.7 & -1.165 \\
\hline 105 & 48 & 16HBE & 46.0 & 1.2 & -40.0 & -1.135 \\
\hline 101 & 48 & $16 \mathrm{HBE}$ & 27.5 & 1.2 & -40.0 & -1.105 \\
\hline 100 & 24 & $16 \mathrm{HBE}$ & 27.5 & 1.2 & -40.0 & -1.026 \\
\hline 102 & 24 & A549 & 27.5 & 1.2 & -40.0 & -0.920 \\
\hline 103 & 48 & A549 & 27.5 & 1.2 & -40.0 & -0.872 \\
\hline 107 & 48 & A549 & 46.0 & 1.2 & -40.0 & -0.844 \\
\hline 106 & 24 & A549 & 46.0 & 1.2 & -40.0 & -0.822 \\
\hline 121 & 24 & $\mathrm{HaCaT}$ & 17.0 & 1.0 & -28.1 & -0.394 \\
\hline 127 & 24 & THP-1 & 100.0 & 1.0 & -32.0 & -0.281 \\
\hline 120 & 24 & A549 & 17.0 & 1.0 & -28.1 & -0.223 \\
\hline 129 & 24 & $\mathrm{HaCaT}$ & 60.0 & 1.0 & -30.6 & -0.197 \\
\hline 128 & 24 & A549 & 60.0 & 1.0 & -30.6 & -0.147 \\
\hline 122 & 24 & NRK-52E & 17.0 & 1.0 & -28.1 & -0.070 \\
\hline 130 & 24 & NRK-52E & 60.0 & 1.0 & -30.6 & 0.059 \\
\hline 123 & 24 & THP-1 & 17.0 & 1.0 & -28.1 & 0.365 \\
\hline 131 & 24 & THP-1 & 60.0 & 1.0 & -30.6 & 0.483 \\
\hline
\end{tabular}

760

761

762

763

764

765

766

767 

is a character which maps a specific value or a range of values. N.B. For brevity, "Correlated descriptors"

771 refers to perfectly correlated descriptors, since the binary descriptors corresponding to the different "Cell type" labels are partially correlated. Only results without correlated descriptors were presented in the main text.

\begin{tabular}{cccc}
\hline \multirow{2}{*}{ Descriptor } & \multirow{2}{*}{ Experimental value } & \multicolumn{3}{c}{ Correlated descriptors } \\
& & Yes & No \\
\hline \multirow{2}{*}{ Treatment Time [h] } & 24 & A & A \\
& 48 & B & No label \\
& 16 HBE & C & C \\
Cell type & A549 & D & D \\
& HaCaT & E & E \\
Average size [nm] & NRK-52E & F & F \\
& THP-1 & G & G \\
Aspect ratio [adimensional] & $\leq 27.5$ & H & No label \\
& $>27.5$ & I & I \\
Zeta potential [mV] & $>1.0$ & J & No label \\
& $>1.0$ & K & K \\
& $>-32.0$ & L & L \\
& $<-32.0$ & M & No label \\
\hline
\end{tabular}

775

776

777

778

779

780

781

782

783

784

785

786

787

788

789

790

791 

on the presence/absence of the relative single character in the original pseudo-SMILES used as input for the CORAL software. Only the approach without correlated descriptors is described.

\begin{tabular}{lccccccccccc}
\hline \multicolumn{1}{c}{ ID } & CORAL: & \multicolumn{1}{c}{ Random Forest: Explicit represtation of descriptors } & \\
& Pseudo-SMILES & A & C & D & E & F & G & I & K & L & pEC 25 \\
\hline 119 & AGK & 1 & 0 & 0 & 0 & 0 & 1 & 0 & 1 & 0 & -1.299 \\
104 & ACIK & 1 & 1 & 0 & 0 & 0 & 0 & 1 & 1 & 0 & -1.272 \\
186 & GK & 0 & 0 & 0 & 0 & 0 & 1 & 0 & 1 & 0 & -1.165 \\
105 & CIK & 0 & 1 & 0 & 0 & 0 & 0 & 1 & 1 & 0 & -1.135 \\
101 & CK & 0 & 1 & 0 & 0 & 0 & 0 & 0 & 1 & 0 & -1.105 \\
100 & ACK & 1 & 1 & 0 & 0 & 0 & 0 & 0 & 1 & 0 & -1.026 \\
102 & ADK & 1 & 0 & 1 & 0 & 0 & 0 & 0 & 1 & 0 & -0.920 \\
103 & DK & 0 & 0 & 1 & 0 & 0 & 0 & 0 & 1 & 0 & -0.872 \\
107 & DIK & 0 & 0 & 1 & 0 & 0 & 0 & 1 & 1 & 0 & -0.844 \\
106 & ADIK & 1 & 0 & 1 & 0 & 0 & 0 & 1 & 1 & 0 & -0.822 \\
121 & AEL & 1 & 0 & 0 & 1 & 0 & 0 & 0 & 0 & 1 & -0.394 \\
127 & AGIL & 1 & 0 & 0 & 0 & 0 & 1 & 1 & 0 & 1 & -0.281 \\
120 & ADL & 1 & 0 & 1 & 0 & 0 & 0 & 0 & 0 & 1 & -0.223 \\
129 & AEIL & 1 & 0 & 0 & 1 & 0 & 0 & 1 & 0 & 1 & -0.197 \\
128 & ADIL & 1 & 0 & 1 & 0 & 0 & 0 & 1 & 0 & 1 & -0.147 \\
122 & AFL & 1 & 0 & 0 & 0 & 1 & 0 & 0 & 0 & 1 & -0.070 \\
130 & AFIL & 1 & 0 & 0 & 0 & 1 & 0 & 1 & 0 & 1 & 0.059 \\
123 & AGL & 1 & 0 & 0 & 0 & 0 & 1 & 0 & 0 & 1 & 0.365 \\
131 & AGIL & 1 & 0 & 0 & 0 & 0 & 1 & 1 & 0 & 1 & 0.483 \\
\hline & & & & & & & & & & & \\
\hline
\end{tabular}

796

797

798

799

800

801

802

803 

test sets for both CORAL and Random Forest approaches. LOO was performed five times using five different

807 training set splits, i.e. five different partitions of a given LOO training set to yield an internal "test set" for 808 hyperparameters' selection, for CORAL and five different seeds for Random Forest. $\mathrm{R}^{2}$ and RMSE values were 809 computed on the predicted values for each training set. Average and standard error of the mean (here reported in 810 brackets) for training set results were calculated over the 19 models - one for each instance in the dataset - for 811 each run of the LOO procedure. These statistics were compared with those obtained from the LOO (i.e. test) 812 predictions. Global results for training sets were calculating by averaging over all the 95 models developed (i.e.

81319 models $\times 5$ LOO runs) whereas, for test sets, global results were obtained by averaging over the five statistics 814 resulting from LOO. N.B. For both methods, only results without perfectly correlated descriptors are presented.

815 For Random Forest, only results without bootstrap sampling are presented.

\begin{tabular}{|c|c|c|c|c|c|}
\hline \multirow{2}{*}{ Software } & \multirow{2}{*}{ Run } & \multicolumn{2}{|c|}{$\mathrm{R}^{2}$} & \multicolumn{2}{|c|}{ RMSE } \\
\hline & & Training set & Test set & Training set & Test set \\
\hline \multirow{6}{*}{ CORAL } & split 1 & $\begin{array}{c}0.8031 \\
(0.0166)\end{array}$ & 0.6529 & $\begin{array}{l}0.2540 \\
(0.0083)\end{array}$ & 0.3443 \\
\hline & split 2 & $\begin{array}{c}0.8567 \\
(0.0056)\end{array}$ & 0.6243 & $\begin{array}{c}0.2176 \\
(0.0085)\end{array}$ & 0.3675 \\
\hline & split 3 & $\begin{array}{c}0.8570 \\
(0.0029)\end{array}$ & 0.6143 & $\begin{array}{c}0.2119 \\
(0.0034)\end{array}$ & 0.3712 \\
\hline & split 4 & $\begin{array}{c}0.7876 \\
(0.0077)\end{array}$ & 0.6436 & $\begin{array}{c}0.2675 \\
(0.0064)\end{array}$ & 0.3441 \\
\hline & split 5 & $\begin{array}{c}0.8383 \\
(0.0108)\end{array}$ & 0.7082 & $\begin{array}{c}0.2222 \\
(0.0067)\end{array}$ & 0.3010 \\
\hline & global & $\begin{array}{c}0.8285 \\
(0.0052)\end{array}$ & $\begin{array}{c}0.6486 \\
(0.0164)\end{array}$ & $\begin{array}{l}0.2347 \\
(0.0038)\end{array}$ & $\begin{array}{c}0.3456 \\
(0.0125)\end{array}$ \\
\hline \multirow{6}{*}{$\begin{array}{l}\text { Random } \\
\text { Forest }\end{array}$} & $\begin{array}{c}\text { seed } \\
1\end{array}$ & $\begin{array}{c}0.8717 \\
(0.0023)\end{array}$ & 0.7741 & $\begin{array}{c}0.2022 \\
(0.0026)\end{array}$ & 0.2646 \\
\hline & $\begin{array}{c}\text { seed } \\
2\end{array}$ & $\begin{array}{c}0.8736 \\
(0.0020)\end{array}$ & 0.7899 & $\begin{array}{c}0.1995 \\
(0.0023)\end{array}$ & 0.2544 \\
\hline & $\begin{array}{c}\text { seed } \\
3\end{array}$ & $\begin{array}{c}0.8725 \\
(0.0022)\end{array}$ & 0.7822 & $\begin{array}{c}0.2015 \\
(0.0025)\end{array}$ & 0.2597 \\
\hline & $\begin{array}{l}\text { seed } \\
4\end{array}$ & $\begin{array}{c}0.8711 \\
(0.0023)\end{array}$ & 0.7707 & $\begin{array}{l}0.2022 \\
(0.0025)\end{array}$ & 0.2665 \\
\hline & $\begin{array}{c}\text { seed } \\
5\end{array}$ & $\begin{array}{c}0.8726 \\
(0.0022)\end{array}$ & 0.7864 & $\begin{array}{l}0.2004 \\
(0.0025)\end{array}$ & 0.2568 \\
\hline & global & $\begin{array}{c}0.8723 \\
(0.0010)\end{array}$ & $\begin{array}{l}0.7807 \\
(0.0036)\end{array}$ & $\begin{array}{l}0.2011 \\
(0.0011)\end{array}$ & $\begin{array}{c}0.2604 \\
(0.0023)\end{array}$ \\
\hline
\end{tabular}


819 Figure 1: Zeta potential versus in vitro cytotoxicity experimental values for each silica nanoparticle in the 820 dataset. The graph shows the gap of toxicological data between -0.822 (ID 106) and -0.394 (ID 121). 821 With the "I" symbols are indicated silica nanoparticles with aspect ratio greater than 1 whereas the "O" symbols refer to nanoparticles with aspect ratio equal to 1. N.B. the labels refer to the descriptors used to encode toxicologically relevant variables for modelling.

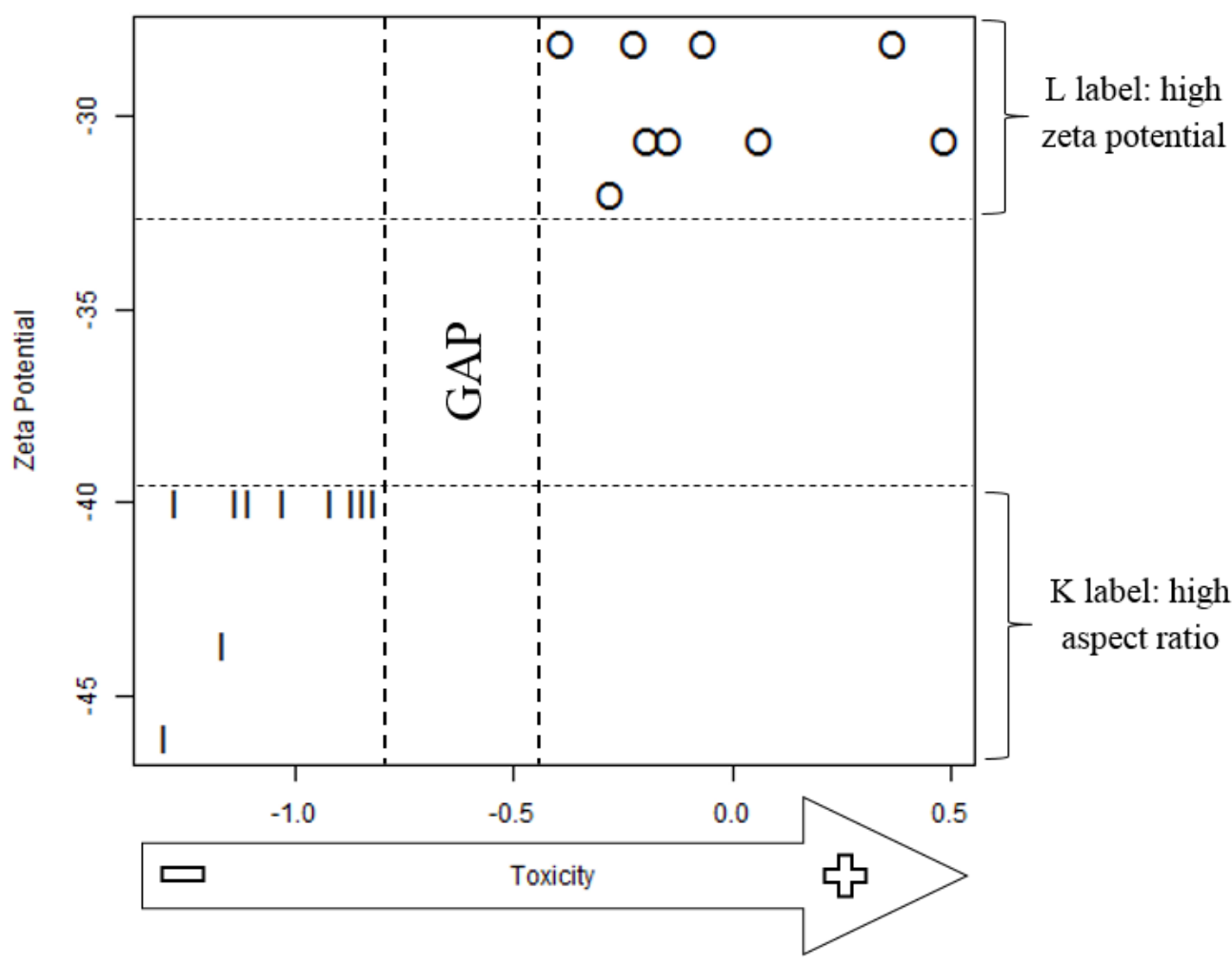

825

826

827

828 

sampling) methods. N.B. (1) All values were scaled to lie between 1 and 0 by dividing by the range (maximum minimum) of values for each variable importance method. (2) Each binary descriptor takes the value 1 or 0 , depending upon the value of the corresponding experimental condition or physico-chemical property. Results were obtained without perfectly correlated descriptors. Error bars represent the standard error of the mean.

841

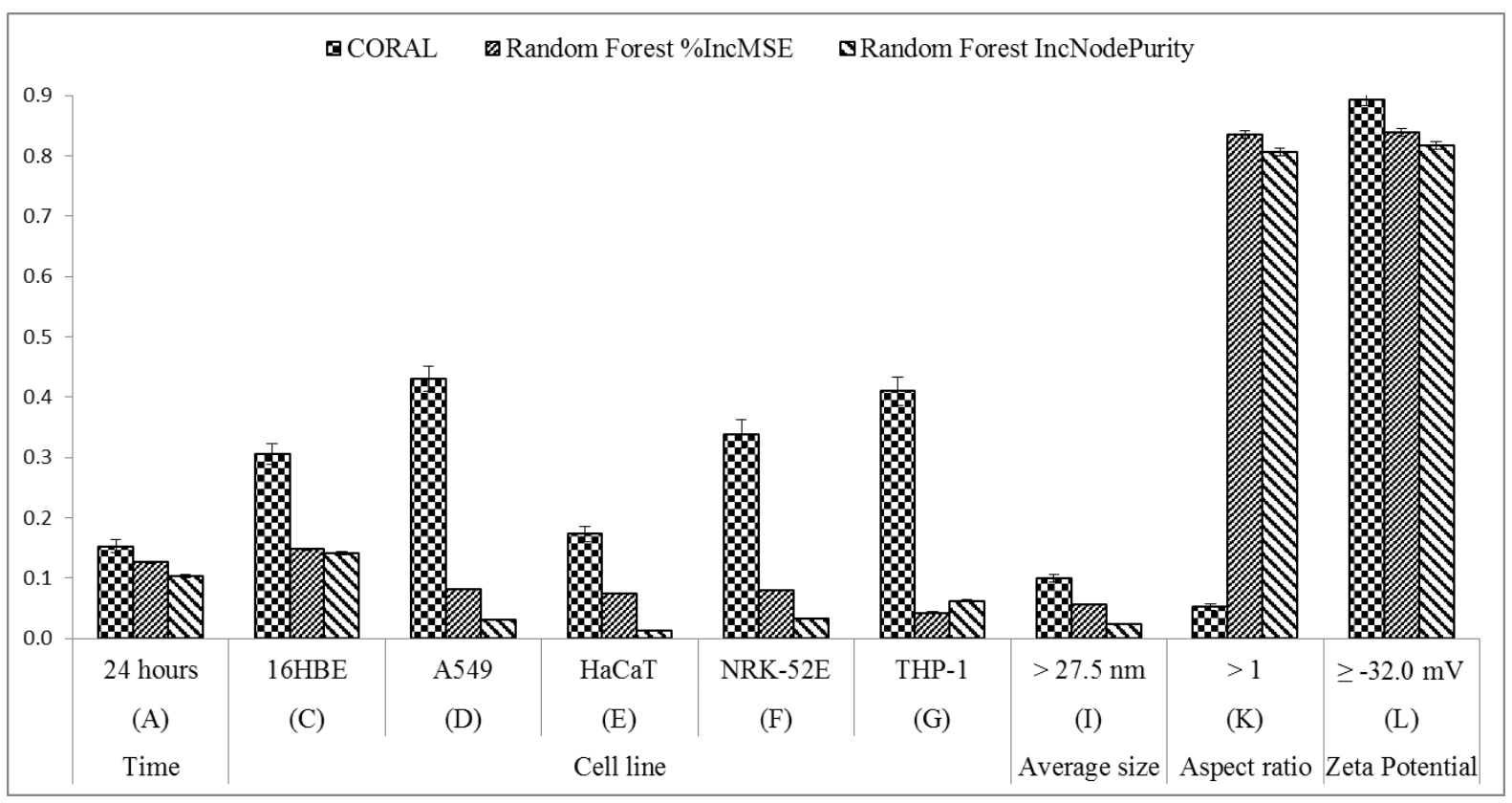

842

843

844

845

846

847

848

849

850

851

852

853

854

855 
Figure 3: Comparison of the feature contribution results for CORAL and Random Forest methods. For

857 CORAL, the "feature contributions" are the correlation weights obtained for a given model built on a given LOO training set. For Random Forest, feature contributions were summarised as pseudocoefficients for a given model built on a given LOO training set. The average value was calculated across all the 95 models developed on LOO training sets. Error bars represent the standard error of For Random Forest, only results without bootstrap sampling are presented.

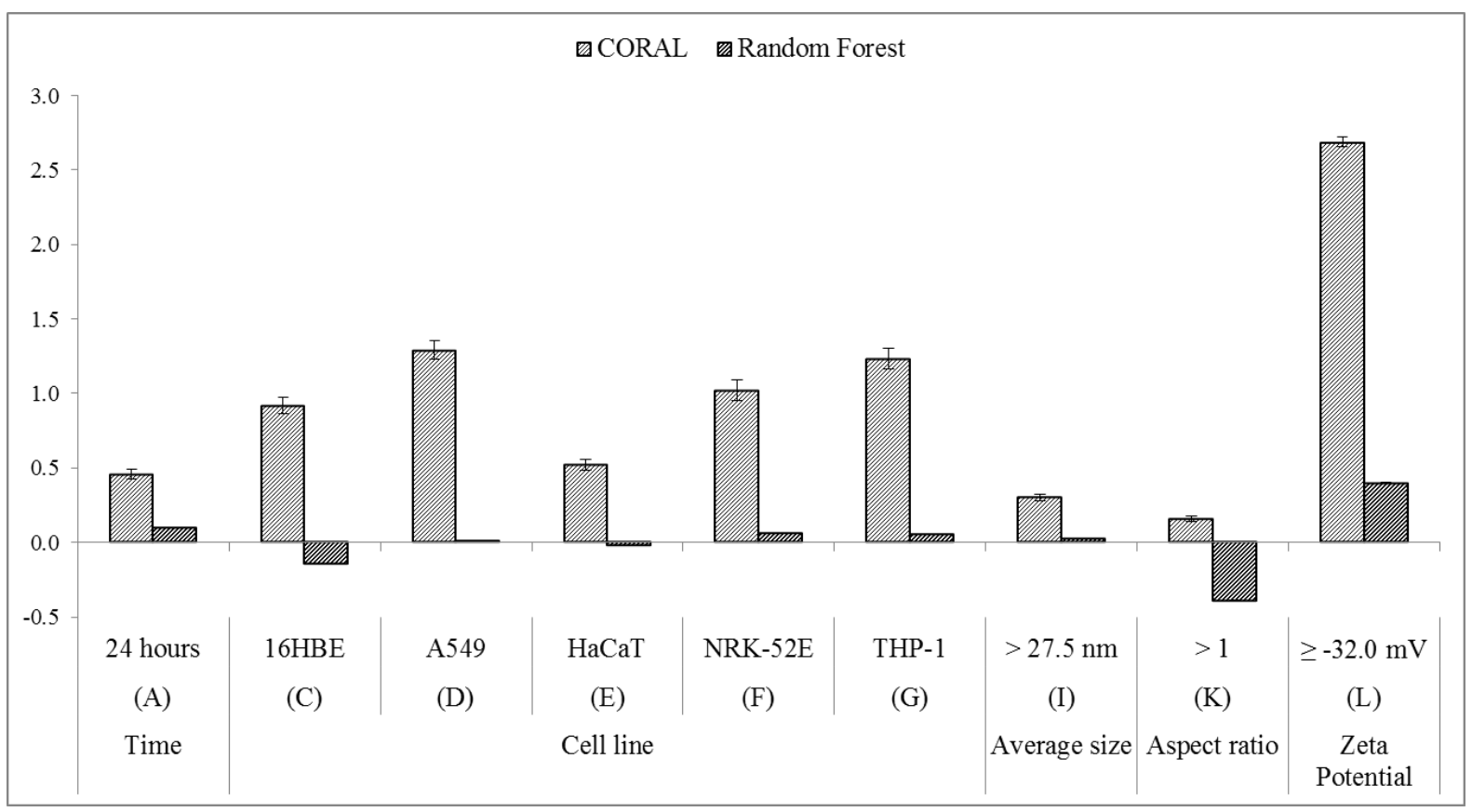




\section{Supplementary Information}

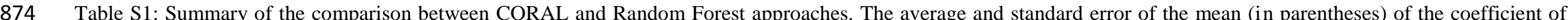

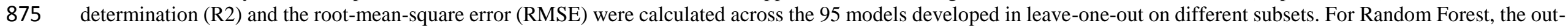

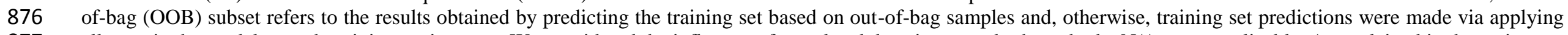

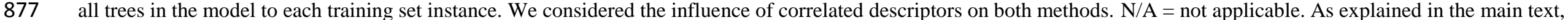

878 "no" correlated descriptors refers to the absence of perfectly correlated descriptors.

\begin{tabular}{|c|c|c|c|c|c|c|c|c|c|c|c|c|c|c|c|c|}
\hline \multirow{2}{*}{ Software } & \multirow{2}{*}{$\begin{array}{l}\text { Correlated } \\
\text { descriptors }\end{array}$} & \multirow{2}{*}{$\begin{array}{l}\text { Bootstrap } \\
\text { sampling }\end{array}$} & \multirow{2}{*}{\multicolumn{2}{|c|}{ LOO subset }} & \multicolumn{2}{|c|}{ Run 1} & \multicolumn{2}{|c|}{ Run 2} & \multicolumn{2}{|c|}{ Run 3} & \multicolumn{2}{|c|}{ Run 4} & \multicolumn{2}{|c|}{ Run 5} & \multicolumn{2}{|c|}{ Global } \\
\hline & & & & & $\mathrm{R}^{2}$ & RMSE & $\mathrm{R}^{2}$ & RMSE & $\mathrm{R}^{2}$ & RMSE & $\mathrm{R}^{2}$ & RMSE & $\mathrm{R}^{2}$ & RMSE & $\mathrm{R}^{2}$ & RMSE \\
\hline \multirow{4}{*}{ CORAL } & \multirow{2}{*}{ Yes } & \multirow{4}{*}{ N/A } & \multicolumn{2}{|c|}{ Training } & $\begin{array}{c}0.8144 \\
(0.0074)\end{array}$ & $\begin{array}{c}0.2519 \\
(0.0057)\end{array}$ & $\begin{array}{c}0.8200 \\
(0.0311)\end{array}$ & $\begin{array}{c}1.0537 \\
(0.8322)\end{array}$ & $\begin{array}{c}0.8528 \\
(0.0037)\end{array}$ & $\begin{array}{c}0.2157 \\
(0.0034)\end{array}$ & $\begin{array}{c}0.7838 \\
(0.0085)\end{array}$ & $\begin{array}{c}0.2688 \\
(0.0066)\end{array}$ & $\begin{array}{c}0.8444 \\
(0.0053)\end{array}$ & $\begin{array}{c}0.2229 \\
(0.0060)\end{array}$ & $\begin{array}{c}0.8231 \\
(0.0071)\end{array}$ & $\begin{array}{c}0.4026 \\
(0.1663)\end{array}$ \\
\hline & & & \multicolumn{2}{|c|}{ Test } & 0.6053 & 0.3916 & 0.0293 & 7.8504 & 0.6465 & 0.3708 & 0.6508 & 0.3336 & 0.6303 & 0.3661 & $\begin{array}{c}0.5124 \\
(0.1210) \\
\end{array}$ & $\begin{array}{c}1.8625 \\
(1.4970) \\
\end{array}$ \\
\hline & \multirow{2}{*}{ No } & & \multicolumn{2}{|c|}{ Training } & $\begin{array}{c}0.8031 \\
(0.0166) \\
\end{array}$ & $\begin{array}{c}0.2540 \\
(0.0083) \\
\end{array}$ & $\begin{array}{c}0.8567 \\
(0.0056)\end{array}$ & $\begin{array}{c}0.2176 \\
(0.0085) \\
\end{array}$ & $\begin{array}{c}0.8570 \\
(0.0029) \\
\end{array}$ & $\begin{array}{c}0.2119 \\
(0.0034) \\
\end{array}$ & $\begin{array}{c}0.7876 \\
(0.0077) \\
\end{array}$ & $\begin{array}{c}0.2675 \\
(0.0064) \\
\end{array}$ & $\begin{array}{c}0.8383 \\
(0.0108) \\
\end{array}$ & $\begin{array}{c}0.2222 \\
(0.0067) \\
\end{array}$ & $\begin{array}{c}0.8285 \\
(0.0052) \\
\end{array}$ & $\begin{array}{c}0.2347 \\
(0.0038) \\
\end{array}$ \\
\hline & & & \multicolumn{2}{|c|}{ Test } & 0.6529 & 0.3443 & 0.6243 & 0.3675 & 0.6143 & 0.3712 & 0.6436 & 0.3441 & 0.7082 & 0.3010 & $\begin{array}{c}0.6486 \\
(0.0164)\end{array}$ & $\begin{array}{c}0.3456 \\
(0.0125)\end{array}$ \\
\hline \multirow{12}{*}{$\begin{array}{c}\text { Random } \\
\text { Forest }\end{array}$} & \multirow{6}{*}{ Yes } & \multirow{3}{*}{ Yes } & \multirow{2}{*}{ Training } & ООВ & $\begin{array}{c}0.7866 \\
(0.0040)\end{array}$ & $\begin{array}{c}0.2533 \\
(0.0030)\end{array}$ & $\begin{array}{c}0.7897 \\
(0.0040)\end{array}$ & $\begin{array}{c}0.2514 \\
(0.0029)\end{array}$ & $\begin{array}{c}0.7884 \\
(0.0032)\end{array}$ & $\begin{array}{c}0.2523 \\
(0.0028)\end{array}$ & $\begin{array}{c}0.7875 \\
(0.0046)\end{array}$ & $\begin{array}{c}0.2526 \\
(0.0033)\end{array}$ & $\begin{array}{c}0.7897 \\
(0.0042)\end{array}$ & $\begin{array}{c}0.2515 \\
(0.0031)\end{array}$ & $\begin{array}{c}0.7884 \\
(0.0018)\end{array}$ & $\begin{array}{c}0.2522 \\
(0.0013)\end{array}$ \\
\hline & & & & Predicted & $\begin{array}{c}0.8985 \\
(0.0025) \\
\end{array}$ & $\begin{array}{c}0.1758 \\
(0.0026) \\
\end{array}$ & $\begin{array}{c}0.9000 \\
(0.0024)\end{array}$ & $\begin{array}{c}0.1743 \\
(0.0026) \\
\end{array}$ & $\begin{array}{c}0.8985 \\
(0.0022) \\
\end{array}$ & $\begin{array}{c}0.1755 \\
(0.0024) \\
\end{array}$ & $\begin{array}{c}0.8991 \\
(0.0026) \\
\end{array}$ & $\begin{array}{l}0.1751 \\
(0.0028) \\
\end{array}$ & $\begin{array}{c}0.9000 \\
(0.0026) \\
\end{array}$ & $\begin{array}{c}0.1744 \\
(0.0027) \\
\end{array}$ & $\begin{array}{c}0.8992 \\
(0.0011) \\
\end{array}$ & $\begin{array}{c}0.1750 \\
(0.0012) \\
\end{array}$ \\
\hline & & & \multicolumn{2}{|c|}{ Test } & 0.7981 & 0.2468 & 0.7996 & 0.2459 & 0.7959 & 0.2481 & 0.7940 & 0.2494 & 0.7901 & 0.2519 & $\begin{array}{c}0.7955 \\
(0.0017) \\
\end{array}$ & $\begin{array}{c}0.2484 \\
(0.0010) \\
\end{array}$ \\
\hline & & \multirow{3}{*}{ No } & \multirow{2}{*}{ Training } & ООВ & $\begin{array}{c}0.7869 \\
(0.0032)\end{array}$ & $\begin{array}{c}0.2530 \\
(0.0028)\end{array}$ & $\begin{array}{c}0.7911 \\
(0.0032)\end{array}$ & $\begin{array}{c}0.2504 \\
(0.0026)\end{array}$ & $\begin{array}{c}0.7932 \\
(0.0035)\end{array}$ & $\begin{array}{c}0.2491 \\
(0.0027)\end{array}$ & $\begin{array}{c}0.7894 \\
(0.0032)\end{array}$ & $\begin{array}{c}0.2515 \\
(0.0027)\end{array}$ & $\begin{array}{c}0.7919 \\
(0.0030)\end{array}$ & $\begin{array}{c}0.2500 \\
(0.0027)\end{array}$ & $\begin{array}{c}0.7905 \\
(0.0014)\end{array}$ & $\begin{array}{c}0.2508 \\
(0.0012)\end{array}$ \\
\hline & & & & Predicted & $\begin{array}{c}0.8732 \\
(0.0018) \\
\end{array}$ & $\begin{array}{c}0.1961 \\
(0.0022) \\
\end{array}$ & $\begin{array}{c}0.8743 \\
(0.0021) \\
\end{array}$ & $\begin{array}{c}0.1953 \\
(0.0022) \\
\end{array}$ & $\begin{array}{c}0.8742 \\
(0.0021) \\
\end{array}$ & $\begin{array}{c}0.1953 \\
(0.0023) \\
\end{array}$ & $\begin{array}{c}0.8752 \\
(0.0021) \\
\end{array}$ & $\begin{array}{c}0.1944 \\
(0.0022) \\
\end{array}$ & $\begin{array}{c}0.8743 \\
(0.0020) \\
\end{array}$ & $\begin{array}{c}0.1953 \\
(0.0023) \\
\end{array}$ & $\begin{array}{c}0.8742 \\
(0.0009) \\
\end{array}$ & $\begin{array}{c}0.1953 \\
(0.0010) \\
\end{array}$ \\
\hline & & & \multicolumn{2}{|c|}{ Test } & 0.7857 & 0.2542 & 0.7896 & 0.2519 & 0.7927 & 0.2500 & 0.7920 & 0.2505 & 0.7977 & 0.2470 & $\begin{array}{c}0.7915 \\
(0.0020)\end{array}$ & $\begin{array}{c}0.2507 \\
(0.0012)\end{array}$ \\
\hline & \multirow{6}{*}{ No } & \multirow{3}{*}{ Yes } & \multirow{2}{*}{ Training } & OOB & $\begin{array}{c}0.7792 \\
(0.0047)\end{array}$ & $\begin{array}{c}0.2594 \\
(0.0032)\end{array}$ & $\begin{array}{c}0.7805 \\
(0.0043)\end{array}$ & $\begin{array}{c}0.2591 \\
(0.0028)\end{array}$ & $\begin{array}{c}0.7819 \\
(0.0051)\end{array}$ & $\begin{array}{c}0.2574 \\
(0.0035)\end{array}$ & $\begin{array}{c}0.7801 \\
(0.0040)\end{array}$ & $\begin{array}{c}0.2591 \\
(0.0030)\end{array}$ & $\begin{array}{c}0.7772 \\
(0.0042)\end{array}$ & $\begin{array}{c}0.2606 \\
(0.0031)\end{array}$ & $\begin{array}{c}0.7798 \\
(0.0020)\end{array}$ & $\begin{array}{c}0.2591 \\
(0.0014)\end{array}$ \\
\hline & & & & Predicted & $\begin{array}{c}0.8989 \\
(0.0026)\end{array}$ & $\begin{array}{c}0.1795 \\
(0.0027) \\
\end{array}$ & $\begin{array}{c}0.8989 \\
(0.0024)\end{array}$ & $\begin{array}{c}0.1793 \\
(0.0025) \\
\end{array}$ & $\begin{array}{c}0.8993 \\
(0.0026) \\
\end{array}$ & $\begin{array}{c}0.1782 \\
(0.0027)\end{array}$ & $\begin{array}{c}0.8990 \\
(0.0024)\end{array}$ & $\begin{array}{c}0.1789 \\
(0.0025)\end{array}$ & $\begin{array}{c}0.8985 \\
(0.0025) \\
\end{array}$ & $\begin{array}{c}0.1792 \\
(0.0026)\end{array}$ & $\begin{array}{c}0.8989 \\
(0.0011) \\
\end{array}$ & $\begin{array}{c}0.1790 \\
(0.0011)\end{array}$ \\
\hline & & & \multicolumn{2}{|c|}{ Test } & 0.7757 & 0.2617 & 0.7896 & 0.2544 & 0.7744 & 0.2622 & 0.7900 & 0.2538 & 0.7867 & 0.2559 & $\begin{array}{c}0.7833 \\
(0.0034) \\
\end{array}$ & $\begin{array}{c}0.2576 \\
(0.0018) \\
\end{array}$ \\
\hline & & \multirow{3}{*}{ No } & \multirow{2}{*}{ Training } & ООВ & $\begin{array}{c}0.7732 \\
(0.0049)\end{array}$ & $\begin{array}{c}0.2644 \\
(0.0038)\end{array}$ & $\begin{array}{c}0.7854 \\
(0.0030)\end{array}$ & $\begin{array}{c}0.2563 \\
(0.0026)\end{array}$ & $\begin{array}{c}0.7737 \\
(0.0030)\end{array}$ & $\begin{array}{c}0.2641 \\
(0.0028)\end{array}$ & $\begin{array}{c}0.7723 \\
(0.0042)\end{array}$ & $\begin{array}{c}0.2642 \\
(0.0034)\end{array}$ & $\begin{array}{c}0.7717 \\
(0.0039)\end{array}$ & $\begin{array}{c}0.2639 \\
(0.0031)\end{array}$ & $\begin{array}{c}0.7753 \\
(0.0018)\end{array}$ & $\begin{array}{c}0.2626 \\
(0.0014)\end{array}$ \\
\hline & & & & Predicted & $\begin{array}{c}0.8717 \\
(0.0023) \\
\end{array}$ & $\begin{array}{c}0.2022 \\
(0.0026)\end{array}$ & $\begin{array}{c}0.8736 \\
(0.0020)\end{array}$ & $\begin{array}{c}0.1995 \\
(0.0023) \\
\end{array}$ & $\begin{array}{c}0.8725 \\
(0.0022) \\
\end{array}$ & $\begin{array}{c}0.2015 \\
(0.0025) \\
\end{array}$ & $\begin{array}{c}0.8711 \\
(0.0023) \\
\end{array}$ & $\begin{array}{c}0.2022 \\
(0.0025) \\
\end{array}$ & $\begin{array}{c}0.8726 \\
(0.0022) \\
\end{array}$ & $\begin{array}{c}0.2004 \\
(0.0025) \\
\end{array}$ & $\begin{array}{c}0.8723 \\
(0.0010) \\
\end{array}$ & $\begin{array}{c}0.2011 \\
(0.0011)\end{array}$ \\
\hline & & & $\mathrm{T}$ & est & 0.7741 & 0.2646 & 0.7899 & 0.2544 & 0.7822 & 0.2597 & 0.7707 & 0.2665 & 0.7864 & 0.2568 & $\begin{array}{c}0.7807 \\
(0.0036)\end{array}$ & $\begin{array}{c}0.2604 \\
(0.0023)\end{array}$ \\
\hline
\end{tabular}


881 error of the mean (SEM) - here reported as error bar - were calculated across all the models developed by LOO. Perfectly correlated descriptors were deleted.

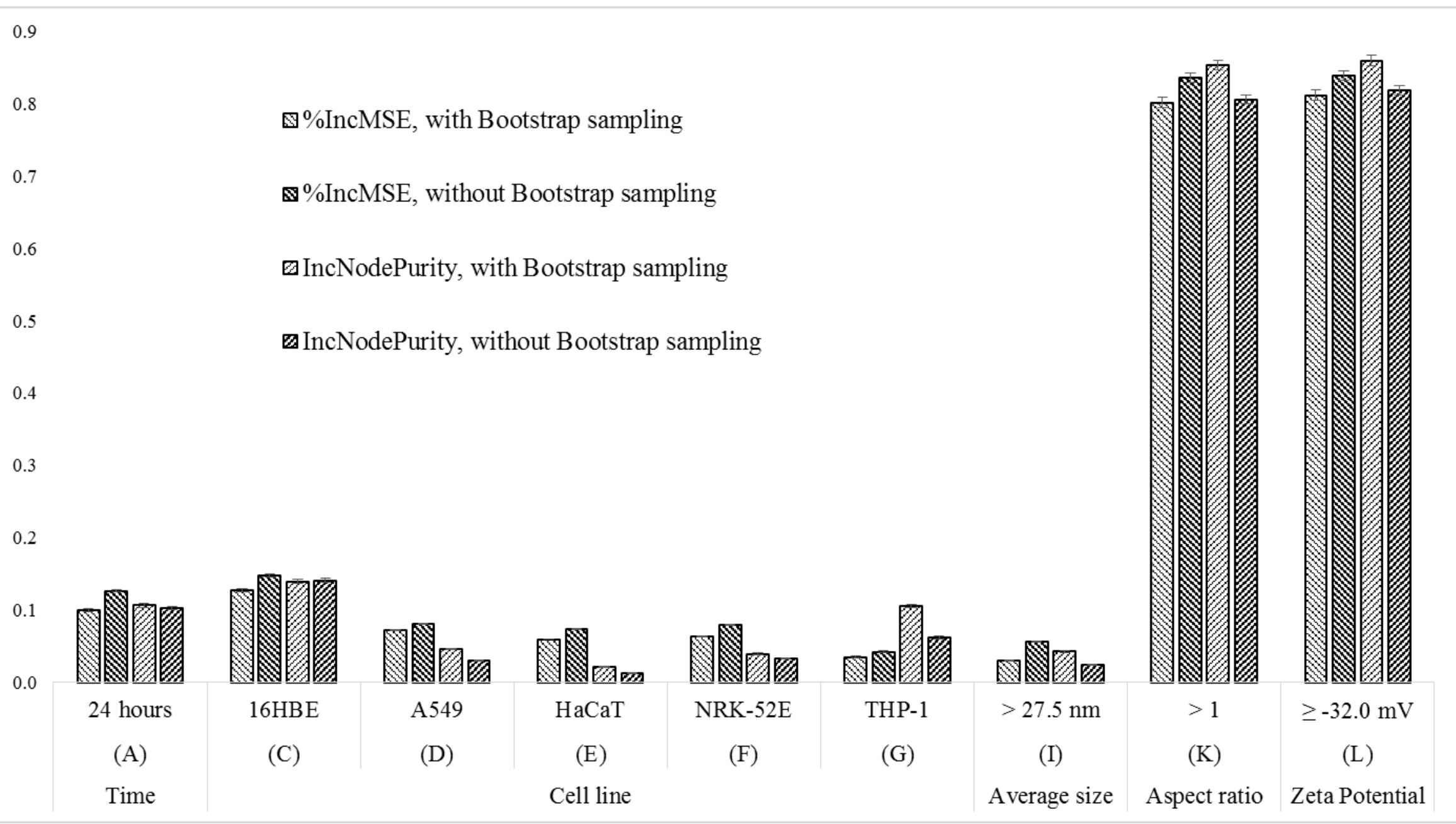




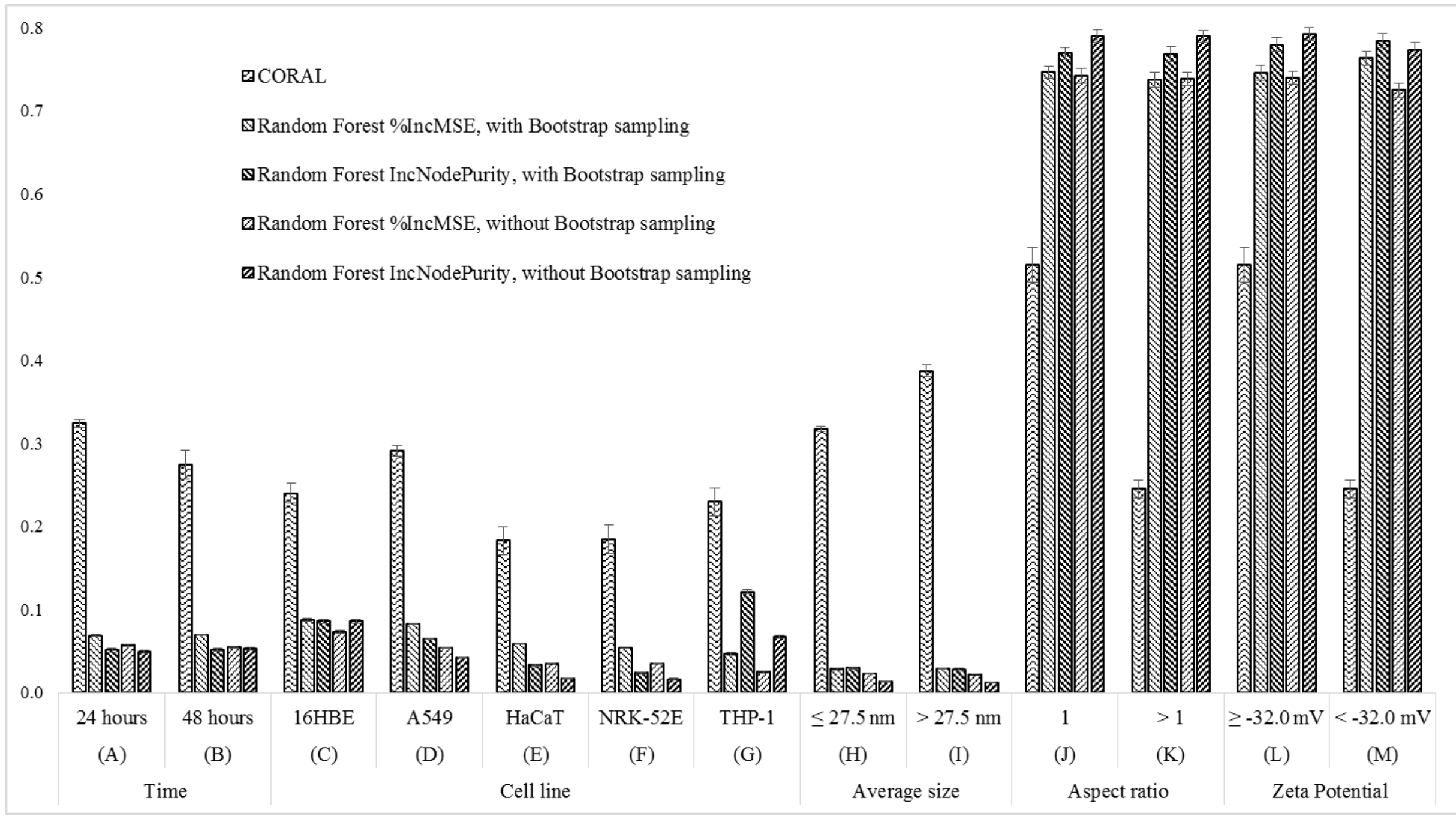


Figure S3: Feature contribution analysis for CORAL and Random Forest methods with correlated descriptors. Error bars represent the standard error of the mean.

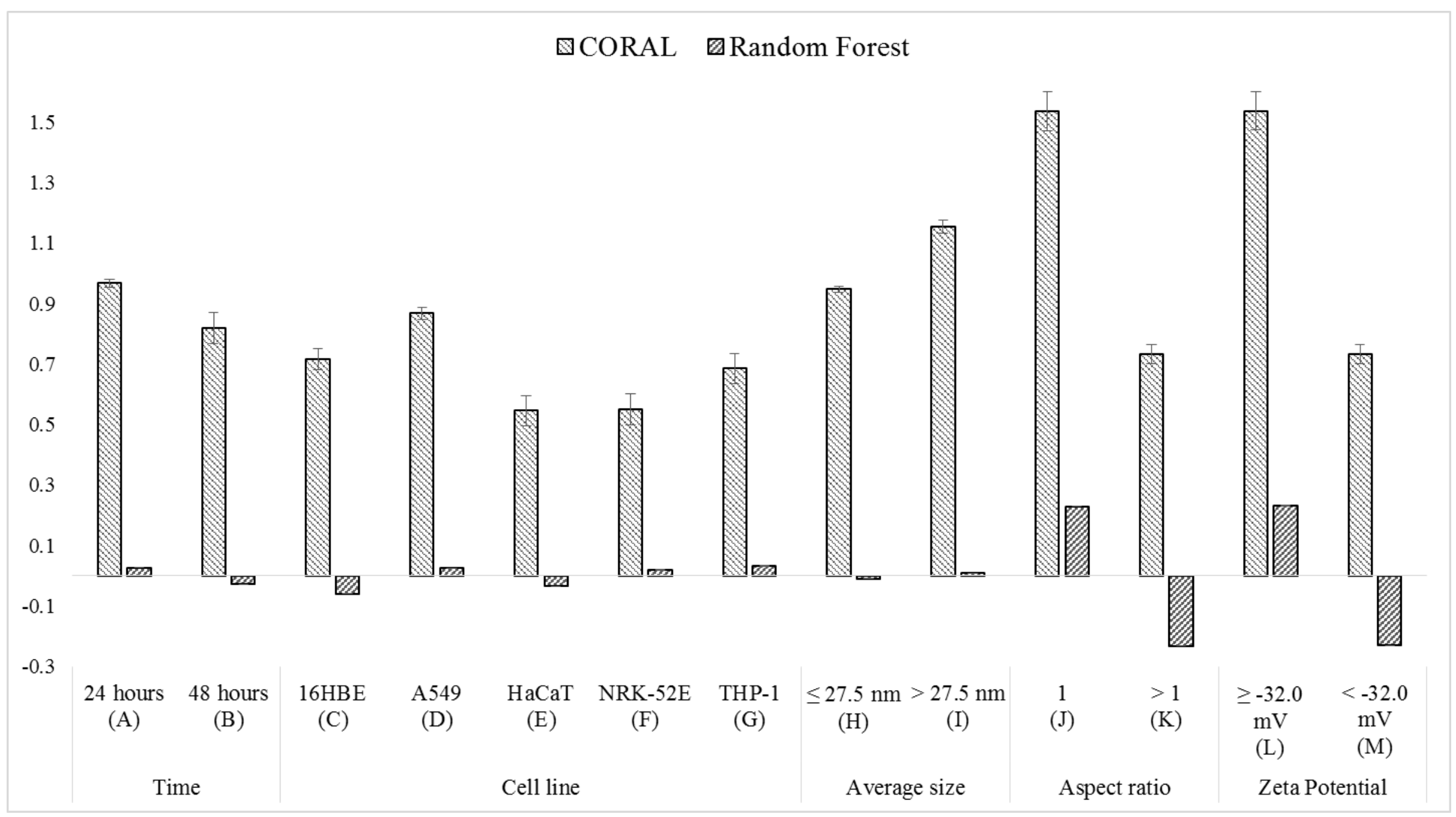


890 As reported in the CORAL software documentation (version: December 17, 2014 for Microsoft Windows, 891 available at http://www.insilico.eu/coral/), in order to use the software, specific text files must be prepared as 892 input. The CORAL software requires the dataset used for model development to be split into three subsets, each 893 of which is labelled with a different character in the input file, termed "sub-training” ("+"), "calibration” (“-“) and 894 “test" (“\#”) subsets. (Since the model hyperparameters may be optimised based upon performance on this "test" 895 set, it may be considered an internal "test" set.) Each of these must contain a minimum number of 3 instances 896 having a similar experimental range in order for the software to work properly. The exact CORAL settings we 897 used in this work are shown in Figure S4. In this work we applied the "additive scheme" for which the optimal 898 descriptor DCW is calculated by summing the correlation weights CWs of each single attribute $S_{k}$ which is present 899 in the input pseudo-SMILES (see main text). Moreover, we selected the "classic scheme" which doesn't use the 900 "calibration" subset. The input files we used do contain instances with the "“-" label for calibration subset but this 901 label was automatically converted by the software into the "+" label for the sub-training subset (see input and 902 output files in Supplementary Information). We applied the recommended approach [40, 42] of optimising the 903 CORAL hyperparameters (i.e. the threshold and number of epochs, "T" and "N") on the internal test set i.e. 904 instances labelled with “\#” (see input files). According to the recommended approach, we prepared five splits of the same input dataset by shuffling the instances between training and test subsets with the rationale of having a similar experimental range among the subsets. Table S2 shows the five different splits we used in this work. For the LOO calculation, a Python script was written to create 19 input text files each containing 18 instances for CORAL modelling (see Supplementary Information). For each time the CORAL software was used for modelling, the single item external test set was predicted using the "Start of DCW and Endpoint Calculation for inserted SMILES” button as shown in Figure S5.

\section{Computational resources used to carry out the calculations}

912 We performed all the calculations, including with Random Forest, on a 32-bit Windows 7 machine with an Intel ${ }^{\circledR}$ Core $^{\mathrm{TM}}$ i3-2120 CPU $3.30 \mathrm{GHz}$ processor and $4 \mathrm{~GB}$ of installed memory (RAM). 
919 Figure S4: CORAL graphical user interface settings used in this work.

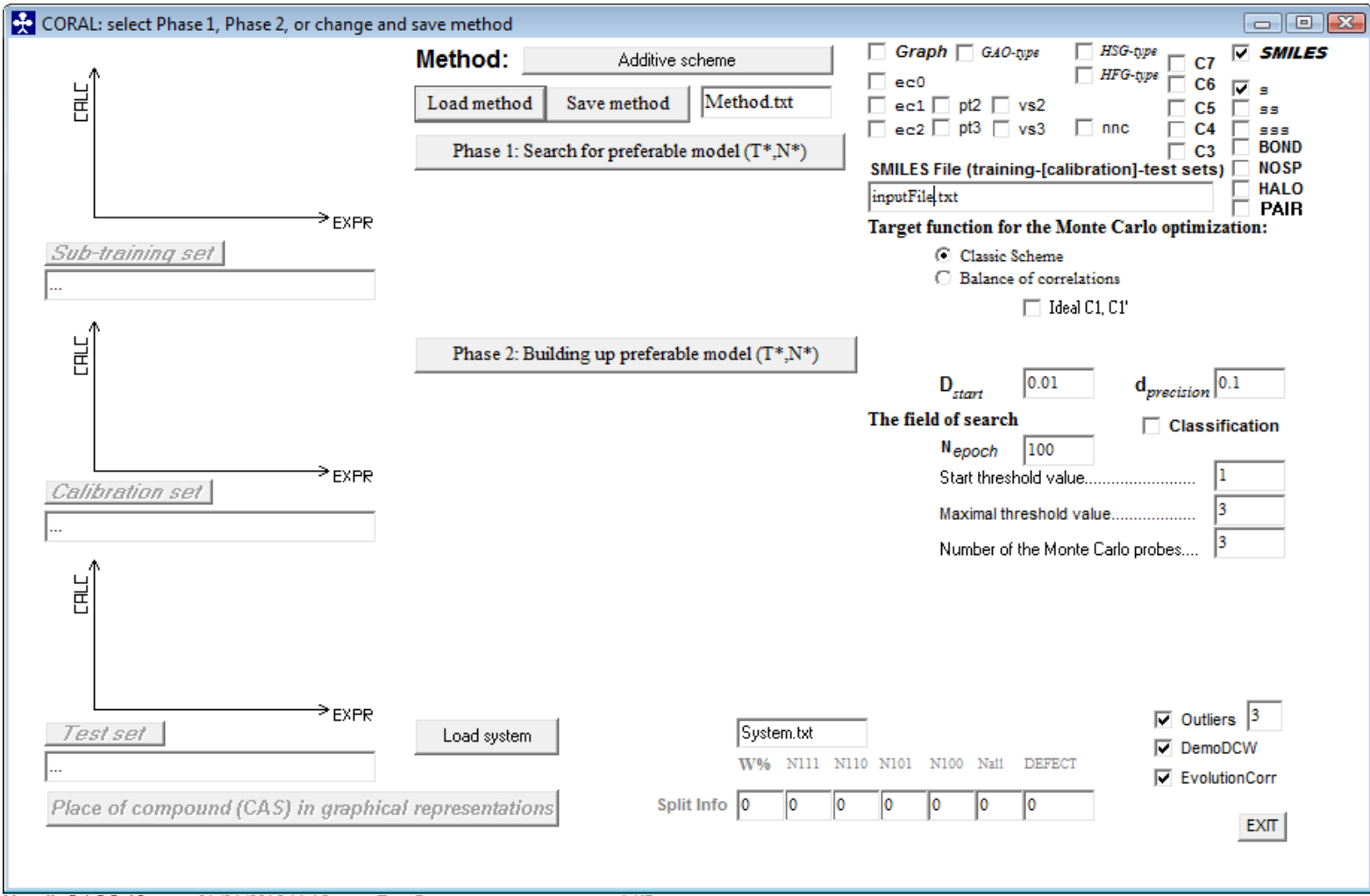


939 Table S2: ID and experimental values (EXP column) for each split of the dataset used for CORAL modelling.

940 N.B. (1) The “+”, “-” and “\#” symbols stand for sub-training, calibration and test subsets, respectively. (2) For

941 "external" LOO validation, one instance was removed at a time and not used for model optimisation, which was

942 performed on the internal "test" subset (“\#”), i.e. each of these splits of the dataset corresponds to a different split

943 of the corresponding LOO training set.

\begin{tabular}{|c|c|c|c|c|c|c|c|c|c|c|}
\hline \multirow{2}{*}{ Subset } & \multicolumn{2}{|c|}{ Split 1} & \multicolumn{2}{|c|}{ Split 2} & \multicolumn{2}{|c|}{ Split 3} & \multicolumn{2}{|c|}{ Split 4} & \multicolumn{2}{|c|}{ Split 5} \\
\hline & ID & EXP & ID & EXP & ID & EXP & ID & EXP & ID & EXP \\
\hline+ & 119 & -1.299 & 119 & -1.299 & 119 & -1.299 & 119 & -1.299 & 119 & -1.299 \\
\hline+ & 105 & -1.135 & 100 & -1.026 & 105 & -1.135 & 105 & -1.135 & 105 & -1.135 \\
\hline+ & 102 & -0.920 & 102 & -0.920 & 102 & -0.920 & 102 & -0.920 & 107 & -0.844 \\
\hline+ & 106 & -0.822 & 106 & -0.822 & 127 & -0.281 & 106 & -0.822 & 106 & -0.822 \\
\hline+ & 120 & -0.223 & 128 & -0.147 & 120 & -0.223 & 120 & -0.223 & 128 & -0.147 \\
\hline+ & 123 & 0.365 & 123 & 0.365 & 123 & 0.365 & 128 & -0.147 & 100 & -1.026 \\
\hline+ & 131 & 0.483 & 131 & 0.483 & 131 & 0.483 & 131 & 0.483 & 131 & 0.483 \\
\hline- & 104 & -1.272 & 104 & -1.272 & 104 & -1.272 & 104 & -1.272 & 104 & -1.272 \\
\hline- & 101 & -1.105 & 127 & -0.281 & 101 & -1.105 & 101 & -1.105 & 101 & -1.105 \\
\hline- & 103 & -0.872 & 107 & -0.844 & 100 & -1.026 & 100 & -1.026 & 103 & -0.872 \\
\hline- & 121 & -0.394 & 121 & -0.394 & 107 & -0.844 & 121 & -0.394 & 121 & -0.394 \\
\hline- & 129 & -0.197 & 129 & -0.197 & 129 & -0.197 & 129 & -0.197 & 129 & -0.197 \\
\hline- & 130 & 0.059 & 130 & 0.059 & 130 & 0.059 & 130 & 0.059 & 130 & 0.059 \\
\hline$\#$ & 186 & -1.165 & 186 & -1.165 & 186 & -1.165 & 186 & -1.165 & 186 & -1.165 \\
\hline$\#$ & 100 & -1.026 & 105 & -1.135 & 103 & -0.872 & 127 & -0.281 & 123 & 0.365 \\
\hline$\#$ & 107 & -0.844 & 103 & -0.872 & 121 & -0.394 & 123 & 0.365 & 102 & -0.920 \\
\hline$\#$ & 127 & -0.281 & 101 & -1.105 & 106 & -0.822 & 103 & -0.872 & 127 & -0.281 \\
\hline$\#$ & 128 & -0.147 & 120 & -0.223 & 128 & -0.147 & 107 & -0.844 & 120 & -0.223 \\
\hline \# & 122 & -0.070 & 122 & -0.070 & 122 & -0.070 & 122 & -0.070 & 122 & -0.070 \\
\hline
\end{tabular}

945

946

947

948

949

950 
952 Figure S5: Screenshot of the CORAL graphical user interface showing an example of calculation of a single item

953 external test set for LOO.

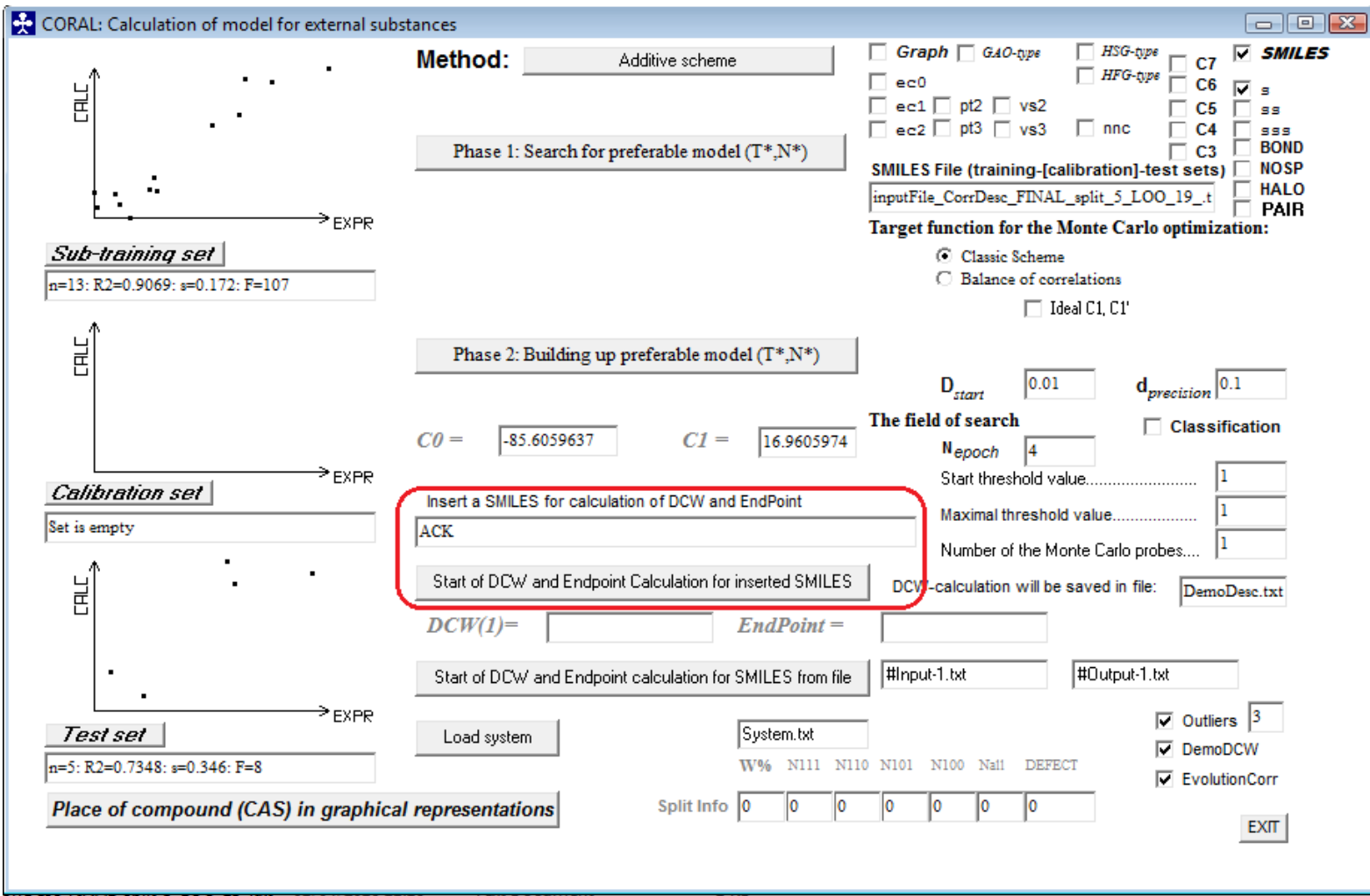

955 